\title{
Type I interferons regulate susceptibility to inflammation-induced preterm birth
}

\author{
Monica Cappelletti, ${ }^{1}$ Pietro Presicce, ${ }^{2}$ Matthew J. Lawson, ${ }^{1,3}$ Vandana Chaturvedi, ${ }^{1,4}$ \\ Traci E. Stankiewicz, ${ }^{1}$ Simone Vanoni, ${ }^{5}$ Isaac T.W. Harley, ${ }^{1}$ Jaclyn W. McAlees, ${ }^{1}$ Daniel A. Giles, ${ }^{1,6}$ \\ Maria E. Moreno-Fernandez, ${ }^{1}$ Cesar M. Rueda, ${ }^{1}$ Paranth Senthamaraikannan, ${ }^{2}$ Xiaofei Sun, ${ }^{7}$ \\ Rebekah Karns, ${ }^{8}$ Kasper Hoebe, ${ }^{1}$ Edith M. Janssen, ${ }^{1}$ Christopher L. Karp, ${ }^{9}$ David A. Hildeman, ${ }^{1}$ \\ Simon P. Hogan, ${ }^{5}$ Suhas G. Kallapur, ${ }^{2}$ Claire A. Chougnet, ${ }^{1}$ Sing Sing Way, ${ }^{4}$ and Senad Divanovic \\ 'Division of Immunobiology, ${ }^{2}$ Division of Neonatology/Pulmonary Biology, Cincinnati Children's Hospital Research \\ Foundation, ${ }^{3}$ Molecular, Cellular and Biochemical Pharmacology Graduate Program, University of Cincinnati College \\ of Medicine, Cincinnati, Ohio, USA. ${ }^{4}$ Division of Infectious Diseases, ${ }^{5}$ Division of Allergy and Immunology, Cincinnat \\ Children's Hospital Research Foundation, and the University of Cincinnati College of Medicine, Cincinnati, Ohio, USA. \\ ${ }^{6}$ Immunology Graduate Program, University of Cincinnati College of Medicine, Cincinnati, Ohio, USA. ${ }^{7}$ Reproductive \\ Sciences, ${ }^{8}$ Castroenterology, Hepatology and Nutrition, Cincinnati Children's Hospital Research Foundation, and the \\ University of Cincinnati College of Medicine, Cincinnati, Ohio, USA. ${ }^{9}$ The Bill \& Melinda Gates Foundation, Seattle, \\ Washington, USA.
}

Preterm birth (PTB) is a leading worldwide cause of morbidity and mortality in infants. Maternal inflammation induced by microbial infection is a critical predisposing factor for PTB. However, biological processes associated with competency of pathogens, including viruses, to induce PTB or sensitize for secondary bacterial infection-driven PTB are unknown. We show that pathogen/ pathogen-associated molecular pattern-driven activation of type I IFN/IFN receptor (IFNAR) was sufficient to prime for systemic and uterine proinflammatory chemokine and cytokine production and induction of PTB. Similarly, treatment with recombinant type I IFNs recapitulated such effects by exacerbating proinflammatory cytokine production and reducing the dose of secondary inflammatory challenge required for induction of PTB. Inflammatory challenge-driven induction of PTB was eliminated by defects in type I IFN, TLR, or IL-6 responsiveness, whereas the sequence of type I IFN sensing by IFNAR on hematopoietic cells was essential for regulation of proinflammatory cytokine production. Importantly, we also show that type I IFN priming effects are conserved from mice to nonhuman primates and humans, and expression of both type I IFNs and proinflammatory cytokines is upregulated in human PTB. Thus, activation of the type I IFN/IFNAR axis in pregnancy primes for inflammation-driven PTB and provides an actionable biomarker and therapeutic target for mitigating PTB risk.

Conflict of interest: The authors have declared that no conflict of interest exists.

License: This work is licensed under the Creative Commons Attribution 4.0 International License. To view a copy of this license, visit http:// creativecommons.org/licenses/by/4.0/

Submitted: October 18, 2016 Accepted: January 17, 2017 Published: March 9, 2017

Reference information: JCI Insight. 2017;2(5):e91288. https:// doi.org/10.1172/jci.insight.91288.

\section{Introduction}

Preterm birth (PTB) is a leading worldwide cause of morbidity and mortality in infants (1). The available diagnostic, therapeutic, and prophylactic armamentarium remains insufficient. Although the precise etiologic basis of PTB is often enigmatic, maternal inflammation has been causally linked to a significant fraction of PTB cases $(1,2)$. In pregnancy, inflammation is physiologically required for uterine contractility, cervical ripening, and the onset of both term birth and PTB $(2,3)$. Pregnant women are at increased risk of PTB during viral infection epidemics (but also during interpandemic periods) (4). In fact, influenza vaccination protects from PTB (5). Notably, several types of pathogens have been detected in the maternal/ fetal tissues of pregnant women with term birth or PTB (6), suggesting that not all pathogens alone cause adverse pregnancy outcomes. However, biological processes associated with competency of pathogens to induce PTB are unknown.

A combined recognition of viral and bacterial molecular patterns by the innate immune receptors (e.g., TLRs), coined a "double-hit hypothesis," has been proposed as a central regulator of increased susceptibility to PTB (7-9). Specifically, viral infection increases the risk of colonization, invasion, and disease due to 
bacterial pathogens (10), and has been linked to sensitization to a secondary bacterial infection-driven PTB (11). Influenza virus, lymphocytic choriomeningitis virus (LCMV), and Listeria species are pathogens commonly associated with induction of PTB (12) and, in addition to other proinflammatory cytokines, drive robust induction of IL-6, TNF, and IFN- $\beta$ (13-25).

Despite significant differences among mammalian species in the biology of pregnancy and parturition, animal models have been highly useful for understanding the molecular mechanisms involved in induction and regulation of birth $(26,27)$. Of note, regulation of immune processes is highly conserved among mammalian species $(28,29)$. In experimental models, challenge with diverse TLR ligands induces cytokine release systemically, in the uterus and fetal membranes (30-32), recruits immune cells into the cervix (33, 34), activates Cox-2 expression (35), and induces contractility, cervical ripening, and PTB (36, 37), while pharmacological inhibition (38) or genetic deletion (39) protects from PTB. Similarly, human studies have shown an association between elevated levels of circulating proinflammatory cytokines and PTB (40, 41). IL-6, in particular, is considered an essential determinant of parturition (42) and a positive predictor of preterm delivery (43).

Notably, however, precisely how subclinical systemic viral infections act as initial inflammatory triggers and increase susceptibility for secondary inflammatory challenge-driven adverse pregnancy outcomes, including PTB, is not well understood. Whether this is unique to viral infections or is conserved among various classes of pathogens known to commonly induce specific inflammatory mediators has not been studied. Further, in this context, the critical immune cells, the specific molecular mediators, their site of expression, and the underlying mechanisms that regulate pathogen-driven susceptibility to inflammationdriven PTB remain undefined. Here we show that: (a) subclinical dose, systemic maternal infection with viral pathogens (e.g., influenza virus or LCMV), or low-dose viral mimic challenge primes for secondary inflammatory challenge-induced PTB; (b) mice with deletion of IL-6, type I IFN receptor (IFNAR), signaling intermediates critical for type I IFN production (e.g., IRF3), or IFN- $\beta$ are protected from inflammation-driven PTB; (c) necessity of IFNAR signaling in priming of secondary inflammatory challenge-driven cytokine production and induction of PTB in mice is conserved across pathogen classes (e.g., Listeria monocytogenes); (d) exogenous type I IFN is sufficient to augment secondary inflammatory challenge-driven inflammatory vigor (e.g., CCL2, IL-6, and TNF production) and to sensitize mice to secondary inflammatory challenge-driven PTB; (e) the sequence of type I IFN priming is essential for regulation of secondary inflammatory challenge-driven inflammatory vigor; (f) type I IFN-mediated regulation of the LPS-driven inflammatory vigor, and specifically priming of IL- 6 and TNF production, is conserved from mice to nonhuman primates and humans; and (g) human PTB patients exhibit increased type I IFN and IL-6 expression. Collectively, the data demonstrate to our knowledge for the first time that activation of the type I IFN/IFNAR axis in pregnancy is critical for regulation of proinflammatory cytokine production and for induction of inflammation-driven PTB via modulation of the host's immune responses.

\section{Results}

Viral infection primes for secondary inflammatory challenge-driven IL-6 production and induction of PTB in mice. Influenza virus and LCMV are pathogens commonly associated with PTB (12) and are known to drive robust production of type I IFNs and proinflammatory cytokines (e.g., IL-6 and TNF) (13-25). We used a tractable double-hit preclinical model of PTB induction for mechanistic insight into how early parturition is triggered (Figure 1A) in the context of a subclinical, systemic viral infection. Notably, the effect of influenza virus infection on pregnancy and tissue tropism has been previously described (44). Low-dose infection with viral pathogens (influenza virus and LCMV; as a mimic of a subclinical infection) sensitized mice to bacterial endotoxin-driven (using LPS, a model of secondary inflammatory challenge) PTB at a dose that, alone, fails to induce PTB (Figure 1A and Supplemental Figure 1A; supplemental material available online with this article; https://doi.org/10.1172/jci.insight.91288DS1). Such viral priming led to significantly increased low-dose LPS-driven type I IFN production, and specifically IFN- $\beta$, as well as IL-6 and TNF proinflammatory cytokine production (Figure 1B and Supplemental Figure 2) and was able to recapitulate the production levels of such mediators seen in context of high-dose LPS challenge. Notably, the effects on cytokine production were identical, irrespective of the data representation method (percentage change or protein concentration; Supplemental Figure 3). To overcome the complexity of a live viral infection, we next used the synthetic TLR3 agonist poly(I:C), a pathogen-associated molecular pattern (PAMP) that molecularly mimics double-stranded RNA commonly produced by viruses, allowing for reductive inter- 
A
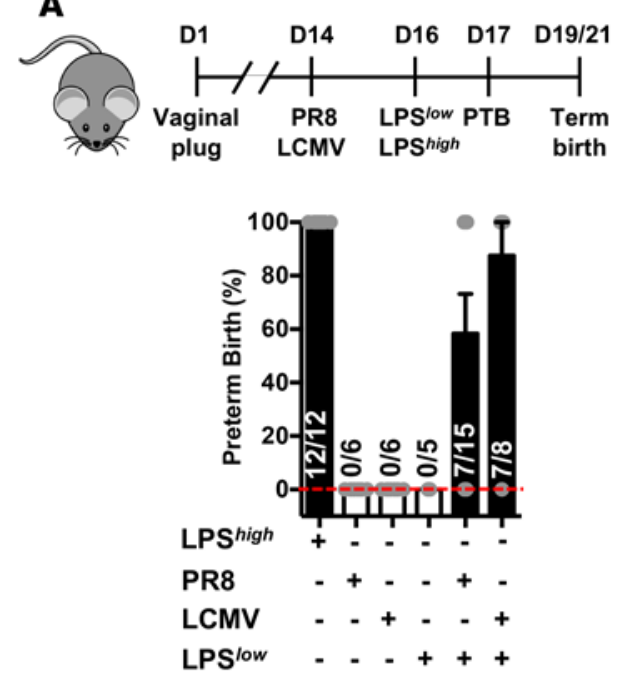

Preterm Birth Term Birth

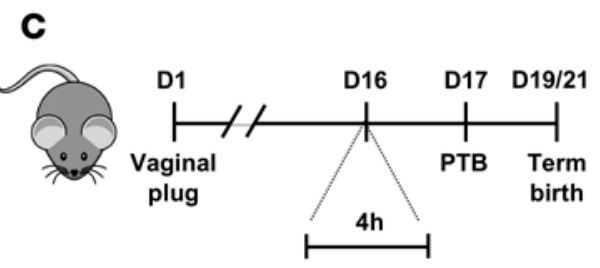

Poly (I:C) Low LPS low Poly (I:C) $)^{\text {high }}$

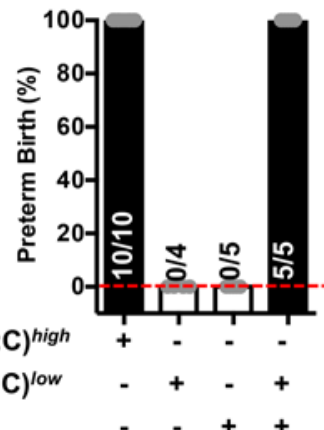

B

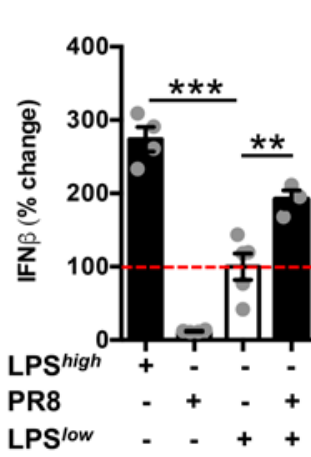

Influenza virus (PR8)
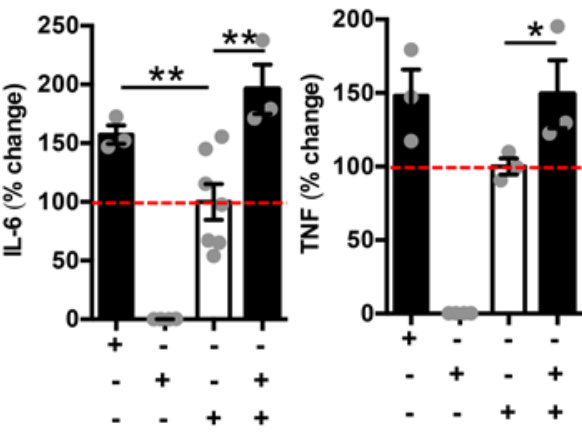

LCMV
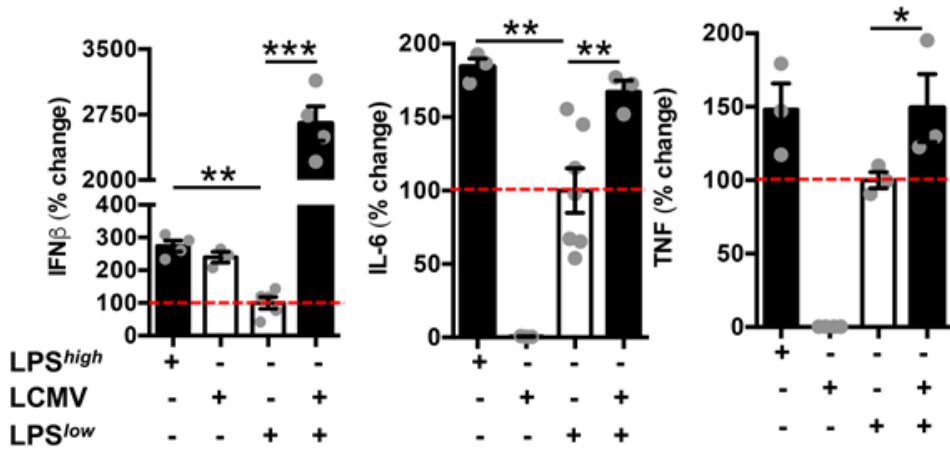

D
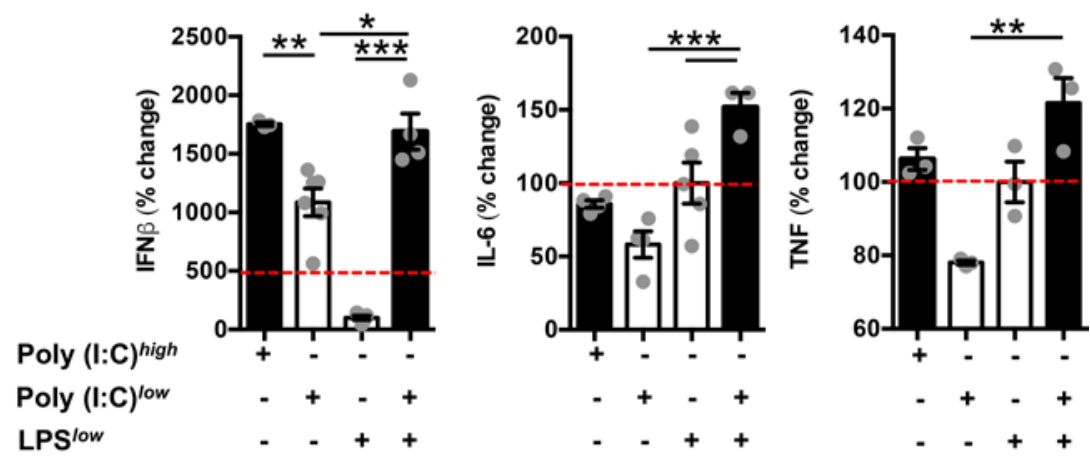

Figure 1. Viral infection primes for secondary inflammatory challenge-driven cytokine production and induction of preterm birth (PTB) in mice. (A) A schematic overview of the tractable double-hit preclinical model of PTB induction employed to define the ability of viral infections to prime for secondary inflammatory challenge-driven PTB. Gravid WT mice were mock infected (saline) or infected with very low dose influenza virus (PR8; $6 \times$ $10^{2} \mathrm{PFU} /$ mouse) or very low dose lymphocytic choriomeningitis virus (LCMV; $5 \times 10^{4} \mathrm{PFU} /$ mouse) on day 14 of gestation prior to being mock challenged (saline) or challenged with LPS ( $25 \mu \mathrm{g} /$ mouse = low; $75 \mu \mathrm{g} /$ mouse $=$ high) on day 16 of gestation, and the incidence of PTB was quantified. (B) WT mice ( $n=3-6 /$ condition) were mock infected or infected as described above in $\mathbf{A}$ with influenza virus or LCMV for 48 hours prior to being mock challenged or challenged with LPS low for 4 hours, and serum IFN- $\beta$, IL-6, and TNF levels were quantified by type I IFN activity assay and in vivo cytokine capture assay (IVCCA), respectively. (C) A schematic overview of the tractable double-hit preclinical model of PTB induction employed to define the ability of viral mimetic to prime for secondary inflammatory challenge-driven PTB. Gravid WT mice were challenged with poly $(\mathrm{I}: \mathrm{C})$ alone $(100 \mu \mathrm{g} / \mathrm{mouse}=\mathrm{low}$; $250 \mu \mathrm{g} /$ mouse $=$ high), or primed with poly $(\mathrm{l}: \mathrm{C})$ for 4 hours prior to being challenged with LPS ${ }^{\text {low }}$ on day 16 of gestation and the incidence of PTB was quantified. (D) WT mice ( $n=3-6 /$ condition) were challenged as described above in $\mathbf{C}$ with poly $(I: C)$ alone or primed with poly $(I: C)$ prior to being challenged with LPS ${ }^{\text {low }}$ for 4 hours and serum IFN- $\beta$, IL-6, and TNF levels were quantified by type I IFN activity assay and IVCCA, respectively. (A and C) Data 
represent percentage induction of PTB. Term birth is defined as parturition on days 19-21 (all pups born alive). PTB is defined as parturition within 24 hours after TLR ligand challenge (all pups deceased). (B and D) Dashed red line represents $100 \%$ induction of cytokine following LPSlow-alone challenge in WT mice. Data (PR8, LCMV, LPS ${ }^{\text {low }}, \mathrm{PR} 8+\mathrm{LPS}^{\text {low }}$, and LCMV $+\mathrm{LPS}^{\text {low }}$ ) represent percentage change over LPS low $(\mathrm{WT}) \pm \mathrm{SEM} .{ }^{*} P<0.05,{ }^{*} P<0.01$, ${ }^{* *} P<0.001$ by ANOVA followed by Tukey's correction.

rogation of underlying mechanisms. As with subclinical systemic viral infection, secondary inflammatory challenge-driven PTB and cytokine production could be mimicked by priming with a low dose of poly(I:C) - a dose that, alone, fails to induce PTB (Supplemental Figure 1B and Figure 1, C and D).

IFNAR signaling is necessary for viral infection-mediated priming of secondary inflammatory challenge-driven cytokine production and induction of PTB in mice. Given the shared induction of type I IFN production after viral infection and TLR signaling activation, and the amplification of type I IFN production via an autocrine/paracrine fashion through the common type I IFN receptor (IFNAR) (45), the necessity of IFNAR in PTB and proinflammatory cytokine production was further investigated. Genetic deletion of IFNAR provided protection against influenza virus- and poly(I:C)-dependent sensitization to secondary LPS challenge-driven PTB (Figure 2A) and was also associated with a 4-fold decrease in IFN- $\beta$, IL-6, and TNF production compared with WT treated mice (Figure 2B and Figure 1, B and D). Similarly, mice with genetic deletion of molecules essential for TLR-mediated activation of IFNAR signaling by poly(I:C), (e.g., TLR3, TRIF, IRF3, and IFN- $\beta$ ) were protected from PTB and exhibited robustly blunted IFN- $\beta$, IL-6, and TNF production induced by high-dose poly(I:C) challenge (Supplemental Figure 4, A and B). As with the poly(I:C) challenge, disruption of type I IFN intermediates and IFNAR resulted in significant protection from high-dose LPS-driven proinflammatory cytokine production and induction of PTB (Supplemental Figure 5). Notably, TLR4-deficient mice were protected from LPS-driven PTB and proinflammatory cytokine production, suggesting the specificity of LPS for the TLR4 signaling pathway (Supplemental Figure 5). However, the protection from high-dose LPS-driven PTB in IFN- $\beta$ - and IFNAR-deficient mice, although significant, was not as robust as that observed with poly(I:C) challenge alone. These data implicate activation of additional signaling pathways in the context of high-dose LPS challenge.

Previous reports have demonstrated that macrophages are central for induction of PTB (46). Similarly, increased chemokine production (e.g., CCL2 and CCL4) has been correlated with macrophage infiltration, activation, tissue inflammation, and induction of PTB (47). Thus, we next examined the contribution of IFNAR signaling to uterine macrophage presence, and chemokine expression. Poly(I:C) challenge of either WT or IFNAR-deficient mice robustly induced uterine CD68 expression (Figure 2C), a well-established marker of macrophage infiltration (48). However, poly(I:C) challenge-driven chemokine expression, and specifically CCL2 expression, was greatly reduced in IFNAR-deficient mice (Figure 2C). Notably, these findings are in agreement with a previous report demonstrating the role of IFNAR signaling in the regulation of lung macrophage infiltration and chemokine expression in the context of Mycobacterium tuberculosis infection (49). Overall, our data suggest that IFNAR signaling may play a role in modulation of uterine macrophage activation

Maternal/fetal interface expression of proinflammatory cytokines has been correlated with induction of parturition and PTB (2). Proinflammatory cytokines, specifically IL-6 and TNF, are robust inducers of Cox-2 expression (50). Cox-2 signaling, which is central for prostaglandin production within intrauterine tissues, is known to play a pivotal role in uterine contractility, cervical ripening, and induction of parturition $(36,37,51,52)$, and has been correlated with induction of PTB in mice and humans $(35,36)$. Thus, we next examined the contribution of IFNAR signaling to poly(I:C) challenge-driven induction of inflammatory (e.g., IL-6 and TNF) and parturition-inducing mediators (e.g., Cox-2) in the reproductive tissues. Poly(I:C) challenge of WT mice resulted in robustly induced uterine IL-6 and TNF expression (Figure 2D) and further correlated with increased uterine Cox-2 expression. Notably, IFNAR ${ }^{-1-}$ mice were fully protected from high-dose poly(I:C)-driven induction of uterine IL-6, TNF, and Cox-2 expression (Figure 2D). These data further suggest that IFNAR signaling, in addition to systemic immune responses, also regulates inflammatory processes in reproductive tissues.

We next examined the functional relevance of dysregulated IL- 6 production via genetic or pharmacological manipulation. As with IFNAR deletion, the genetic deletion of IL-6 provided protection against poly(I:C)- and influenza priming-induced PTB and proinflammatory cytokine production (Figure 2, E and F). Further, antibody-mediated neutralization of IL-6, but not IgG isotype control, protected from poly(I:C)-primed LPS-driven (low-dose) PTB, as well as high-dose poly(I:C)-driven PTB (Figure 2G). 
A

$\underline{\text { IFNAR }^{-1}}$

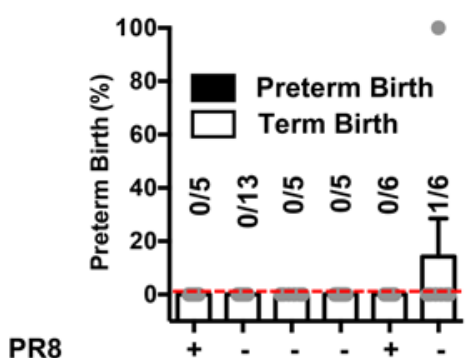

Poly (I:C) high - + - - - -

Poly (I:C) low $\quad-\quad-\quad+\quad-\quad+$

LPS ${ }^{\text {low }}$
B

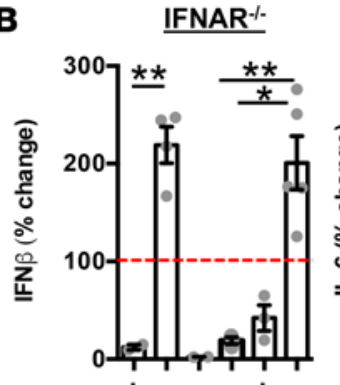

PR8

Poly (I:C) high

Poly (I:C) low

LPS low
IFNAR ${ }^{-1}$
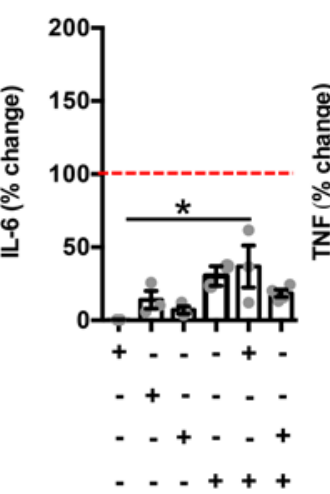

IFNAR ${ }^{-1-}$
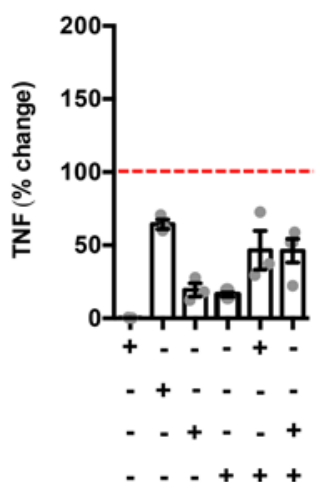

Poly (I:C) high
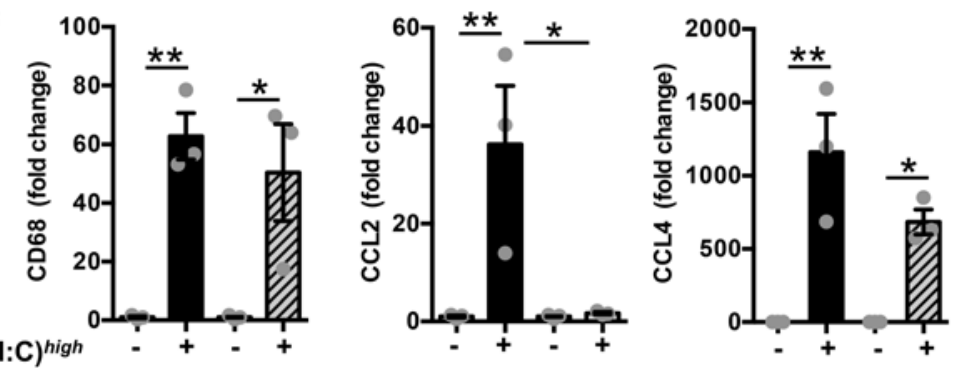

WT

WIIA IFAR ${ }^{-1-}$
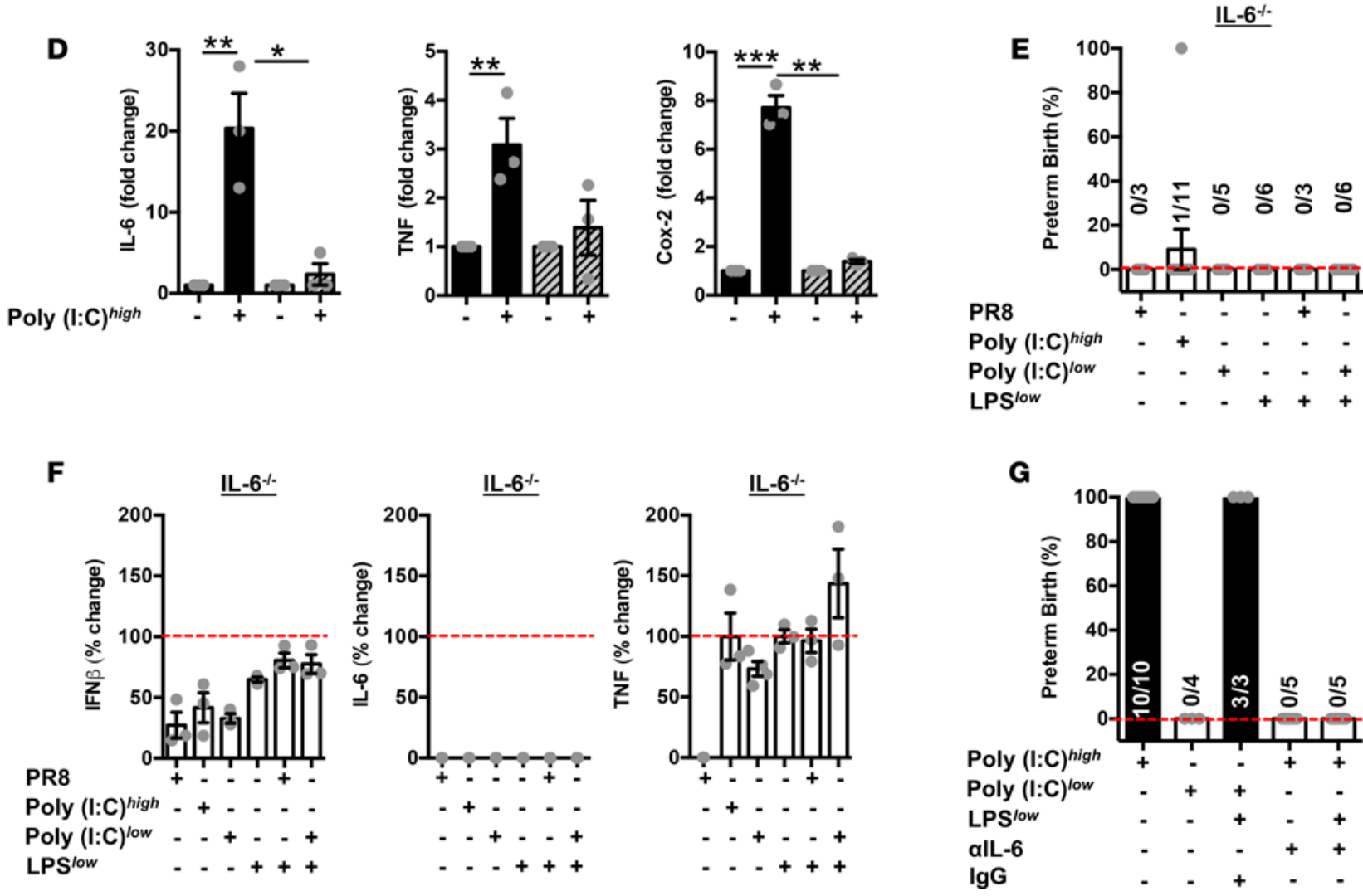

Figure 2. IFNAR signaling is necessary for viral infection-mediated priming of secondary inflammatory challenge-driven cytokine production and induction of preterm birth (PTB) in mice. (A) Gravid type I IFN receptor-deficient (IFNAR ${ }^{-1}$ ) mice were infected with very low dose influenza virus (PR8; 6 $\times 10^{2} \mathrm{PFU} /$ mouse) as described in Figure $1 \mathrm{~A}$ or were challenged with poly $(\mathrm{I}: \mathrm{C})$ alone $(100 \mu \mathrm{g} /$ mouse $=$ low; $250 \mu \mathrm{g} / \mathrm{mouse}=$ high $)$, or primed with poly(l:C $)^{\text {low }}$ for 4 hours prior to being challenged with LPS low $\left(25 \mu \mathrm{g} /\right.$ mouse) on day 16 of gestation, and the incidence of PTB was quantified. (B) IFNAR ${ }^{-/-}$mice $(n=3-6 /$ condition) were infected as described in Figure $1 \mathrm{~A}$ or were challenged with poly $(\mathrm{I}: \mathrm{C})$ alone, or primed with poly $(\mathrm{I}: \mathrm{C})^{\text {low }}$ for 4 hours prior to being challenged with LPS low for 4 hours, and serum IFN- $\beta$, IL-6, and TNF levels were quantified by type I IFN activity assay and in vivo cytokine capture assay (IVCCA), respectively. (C and $\mathbf{D})$ Gravid WT and IFNAR ${ }^{-1-}$ mice were challenged with poly(I:C) ${ }^{\text {high }}$ for 12 hours and uterine CD68, CCL2, CCL4, IL-6, Cox-2, and TNF 
mRNA expression ( $n=3 /$ condition) was quantified. (E) Gravid IL-6 ${ }^{-1-}$ mice were infected with influenza as described in Figure $1 \mathrm{~A}$, or were challenged with poly $(\mathrm{I}: \mathrm{C})$ alone or primed with poly $(\mathrm{I}: \mathrm{C})^{\text {low }}$ for 4 hours prior to being challenged with LPS low on day 16 of gestation, and the incidence of PTB was quantified. (F) IL-6-/- mice ( $n=3 /$ condition) were infected as described in Figure $1 \mathrm{~A}$ or were challenged with poly(I:C) alone, or primed with poly(I:C) ${ }^{\text {low }}$ for 4 hours prior to being challenged with LPS ${ }^{\text {low }}$ for 4 hours, and serum IFN- $\beta$, IL-6, and TNF levels were quantified by type I IFN activity assay and IVCCA, respectively. (G) Gravid WT mice were treated with IL-6-neutralizing Ab or isotype control Ab ( $500 \mu \mathrm{g} /$ mouse) and challenged as described in Figure 1C, and the incidence of PTB was quantified. (A, E, and G) Data represent percentage induction of PTB. (B and G) Dashed red line represents $100 \%$ induction of cytokine following

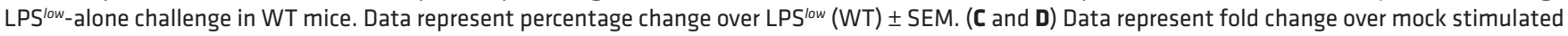
(WT). (B-D and F) ${ }^{*} P<0.05,{ }^{* *} P<0.01,{ }^{* *} P<0.001$ by ANOVA followed by Tukey's correction.

Taken together, these data implicate the type I IFN/IFNAR axis as a modulator of induction of both systemic and uterine proinflammatory cytokines and their downstream mediators, and correlate both the IFNAR and IL- 6 axes with the pathogenesis of inflammation-induced PTB.

Necessity of IFNAR signaling in priming of secondary inflammatory challenge-driven cytokine production and induction of PTB in mice is conserved across pathogen class. We next examined whether these IFNAR and IL-6 axes events were specific to viral infection (or its reductive mimics). Listeria species have been associated with induction of PTB (12) and are known to drive robust induction of IFN- $\beta$, IL-6, and TNF (13-23). The effect of Listeria infection on pregnancy and tissue tropism has been previously described (44). As observed in the context of viral infection or viral mimetic priming, low-dose infection with L. monocytogenes (Lm; as a mimic of a subclinical infection), a pathogen associated with adverse pregnancy outcomes (12), that alone failed to induce PTB, sensitized WT mice to secondary inflammatory challenge-driven PTB and proinflammatory cytokine production (Figure 3, A and B). As the pathogenicity of $L m$ is correlated with its ability to induce type I IFNs (53), we infected mice with an attenuated $\mathrm{Lm}$ strain lacking listeriolysin $\mathrm{O}$ (LLO) and phosphatidylinositol-specific phospholipase C (PLC) that is unable to gain access to the cell cytoplasm and induce type I IFN production $\left(L m^{\Delta}\right)(54)$. Even high-dose $L m^{\Delta}$ infection (10 $\mathrm{CFU}$ ) failed to sensitize mice to LPS-driven PTB or IFN- $\beta$, IL-6, and TNF production (Figure 3, A and B). Similarly, genetic deletion of IFNAR and IL-6 protected from $L m$-driven sensitization to LPS-driven PTB and proinflammatory cytokine production (Figure 3, C-F). Further, as expected the lack of TLR4 expression did not affect poly(I:C)-driven cytokine production; however, it was found to be essential for proinflammatory cytokine production in the context of subclinical infection-dependent priming of secondary LPS challenge (Supplemental Figure 6). Thus, the functional consequences of activation of the type I IFN/IFNAR/IL-6 axis, in terms of effects on cytokine production and induction of PTB, is not specific to viral infections but is rather conserved across pathogen classes.

IFN- $\beta$ is sufficient to prime for secondary inflammatory challenge-driven cytokine production and induction of PTB in mice. As influenza, LCMV, and Listeria are robust inducers of type I IFNs, we next formally tested whether IFN- $\beta$ was sufficient to prime for LPS-driven PTB. The dose of recombinant IFN- $\beta\left(1 \times 10^{4} \mathrm{U} /\right.$ mouse $)$ employed was chosen based on published reports $(55,56)$ and was found to be within or below the range of IFN- $\beta$ levels induced by the influenza virus, LCMV, or $L m$ infection (Supplemental Figure 7). Whereas exogenous administration of IFN- $\beta$ alone induced neither PTB nor proinflammatory cytokine production, IFN- $\beta$ administration was sufficient to prime WT mice for a secondary challenge low-dose LPS-driven PTB (something dependent on IL-6; Figure 4A) and exacerbate IFN- $\beta$, IL-6, and TNF production to the levels equal to high-dose LPS challenge (Figure 4B). Further, we examined whether IFN- $\beta$ sufficiency to prime for secondary inflammatory challenge-driven PTB correlated with uterine chemokine expression, macrophage presence, inflammation, and induction of mediators linked to parturition. As observed with poly(I:C) challenge (Figure 1C), IFN- $\beta$ priming was sufficient to augment uterine CCL2, CCL4 IL-6, TNF, and Cox-2 expression to the levels equal to high-dose LPS challenge (Figure 4D). These data suggest that IFN- $\beta$ priming is sufficient to lower the necessary threshold for LPS-driven inflammation systemically and at reproductive sites, as well as sensitize for induction of PTB.

Initial type I IFN engagement of IFNAR on hematopoietic cells is required for augmentation of secondary inflammatory challenge-driven cytokine production in mice. In the context of viral or intracellular bacterial infection, the majority of systemic type I IFN production is dependent on activation of myeloid cells (13). However, in the context of IFN- $\beta$ priming of exacerbated secondary LPS-driven proinflammatory cytokine production, the critical type I IFN-responsive cell type(s) has not been defined. Thus, we next examined the contribution of IFNAR expression to hematopoietic or non-hematopoietic cells in this model. Reciprocal bone marrow transfers between WT and IFNAR-deficient mice demonstrated that IFNAR expression by hematopoietic cells is critical for IFN- $\beta$ priming of LPS-driven exacerbation of 
A

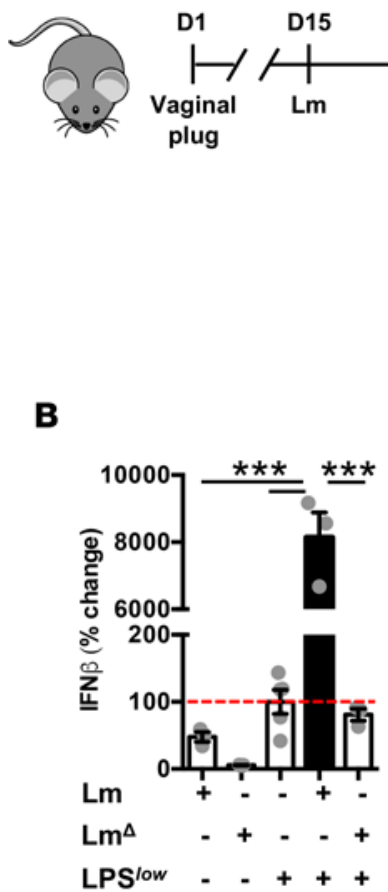

C

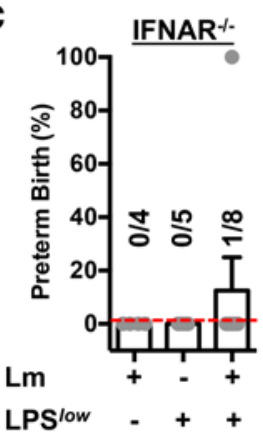

E

IL-6\%

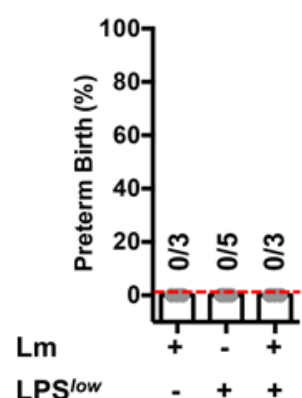

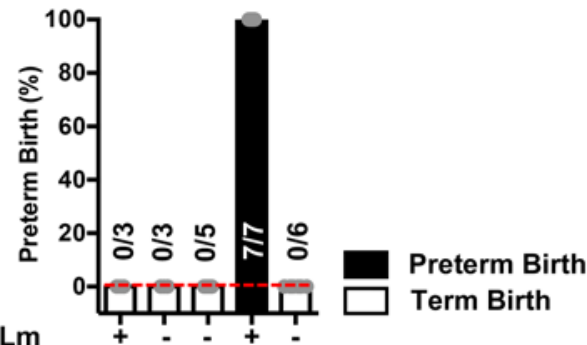

Lm

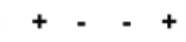

LPS low - - + + +
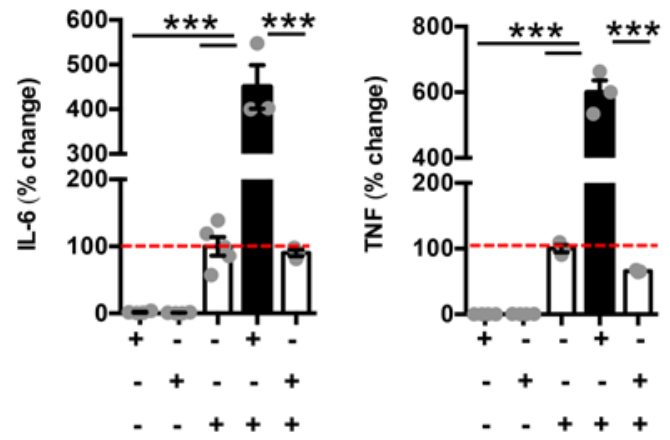

D

IFNAR
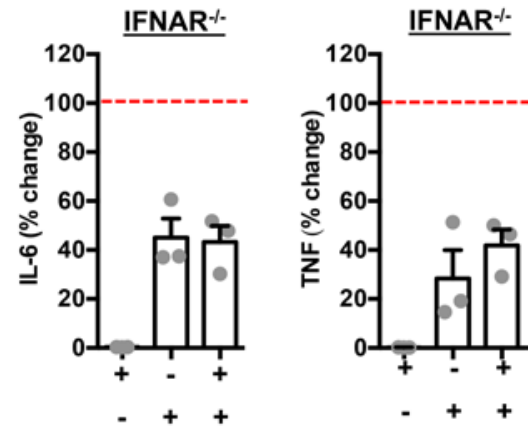

F

$\underline{\mathrm{IL}-6^{-1}}$
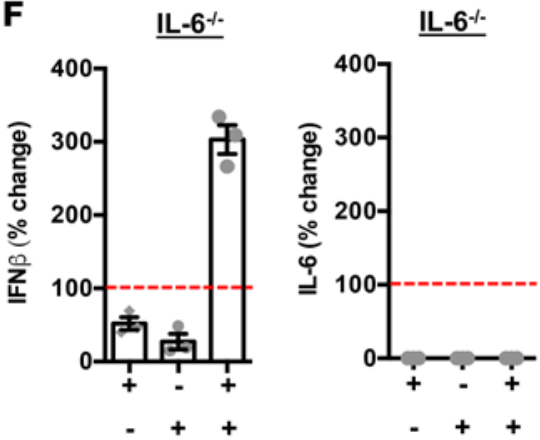

Figure 3. Necessity of IFNAR signaling in priming of secondary inflammatory challenge-driven cytokine production and induction of preterm birth (PTB) in mice is conserved across pathogen class. (A) A schematic overview of the tractable double-hit preclinical model of PTB induction employed to define the ability of Listeria infection to prime for LPS-driven PTB. Gravid WT mice were mock infected (saline) or infected with $L$. monocytogenes $(L m)$ (WT, $1 \times 10^{2}$ CFU; $L^{\Delta}[\Delta$ LLO $\Delta$ PLC $], 1$ $\times 10^{8} \mathrm{CFU}$ ) on day 15 of gestation prior to being mock challenged (saline) or challenged with LPS ${ }^{\text {low }}$ ( $25 \mu \mathrm{g} /$ mouse) on day 16 of gestation, and the incidence of PTB was quantified. (B) WT mice ( $n=3-5 /$ condition) were mock infected or infected with $L m$ (WT and $L m^{\Delta}$ ) for 24 hours prior to being mock challenged or challenged with LPS $^{\text {low }}$ for 4 hours and serum IFN- $\beta$, IL-6, and TNF levels were quantified by type I IFN activity assay and in vivo cytokine capture assay (IVCCA), respectively. (C and $\mathbf{E}$ ) Gravid type I IFN receptor-deficient (IFNAR ${ }^{-/-}$) and $\mathrm{IL}-6^{-/-}$mice were treated as in $\mathbf{A}$ and the incidence of PTB was quantified. (D and F) IFNAR ${ }^{-1-}$ and IL-6- ${ }^{-1-}$ mice ( $n=3-4 /$ condition) were treated as in $\mathbf{B}$ and serum IFN- $\beta$, IL-6, and TNF levels were quantified. (A, C, and $\mathbf{E}$ ) Data represent percentage induction of term birth or PTB. (B, D, and F) Dashed red line represents $100 \%$ induction of cytokine following LPS ${ }^{\text {low }}$ alone challenge in WT mice. Data represent percentage change over $\mathrm{LPS}^{\text {low }}$ $(\mathrm{WT}) \pm \mathrm{SEM}$. ${ }^{* *} P<0.001$ by ANOVA followed by Tukey's correction.

IL-6 and TNF production (Figure $5 \mathrm{~A})$. We next analyzed the sufficiency of such an effect ex vivo and have begun to identify the systemic immune cells types largely responsible for augmented proinflammatory cytokine production. Notably, within the peripheral blood mononuclear cell (PBMC) pool, myeloid cells

$\left(\mathrm{CD} 11 \mathrm{~b}^{+} \mathrm{GR} 1^{-}\right)$represented the most pronounced contributor to exacerbated IL- 6 and TNF production (Figure 5B). These findings correlate with our findings of increased systemic and uterine chemokine and proinflammatory cytokine levels (Figure 4).

As viral infection has been linked to sensitization to a secondary bacterial infection-driven PTB (11), we next examined the sequence and duration of IFN- $\beta$ priming of proinflammatory cytokine production. Markedly, only initial IFN- $\beta$ priming was sufficient to exacerbate secondary inflammatory challenge-driven IL-6 and TNF production, both in vivo (Figure 5C) and in vitro (Figure 5D) - something that is dependent on IFNAR expression. Further, the extent of IFN- $\beta$ priming effects on IL- 6 production in vivo 
A

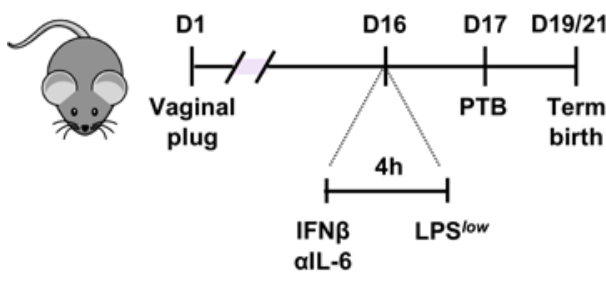

B

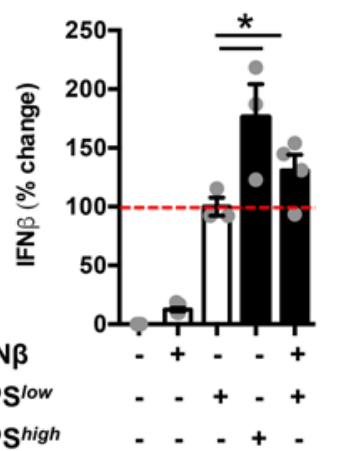

C

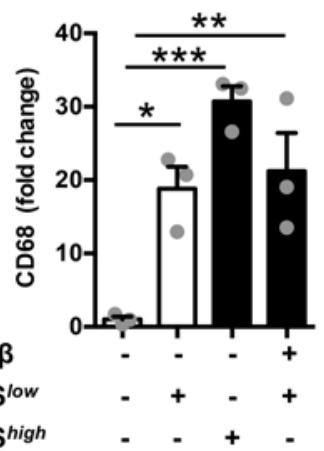

D

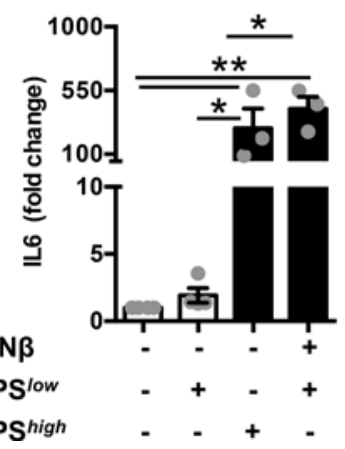

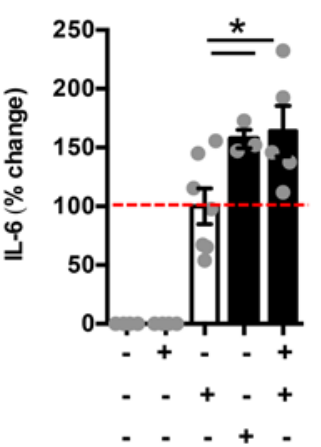
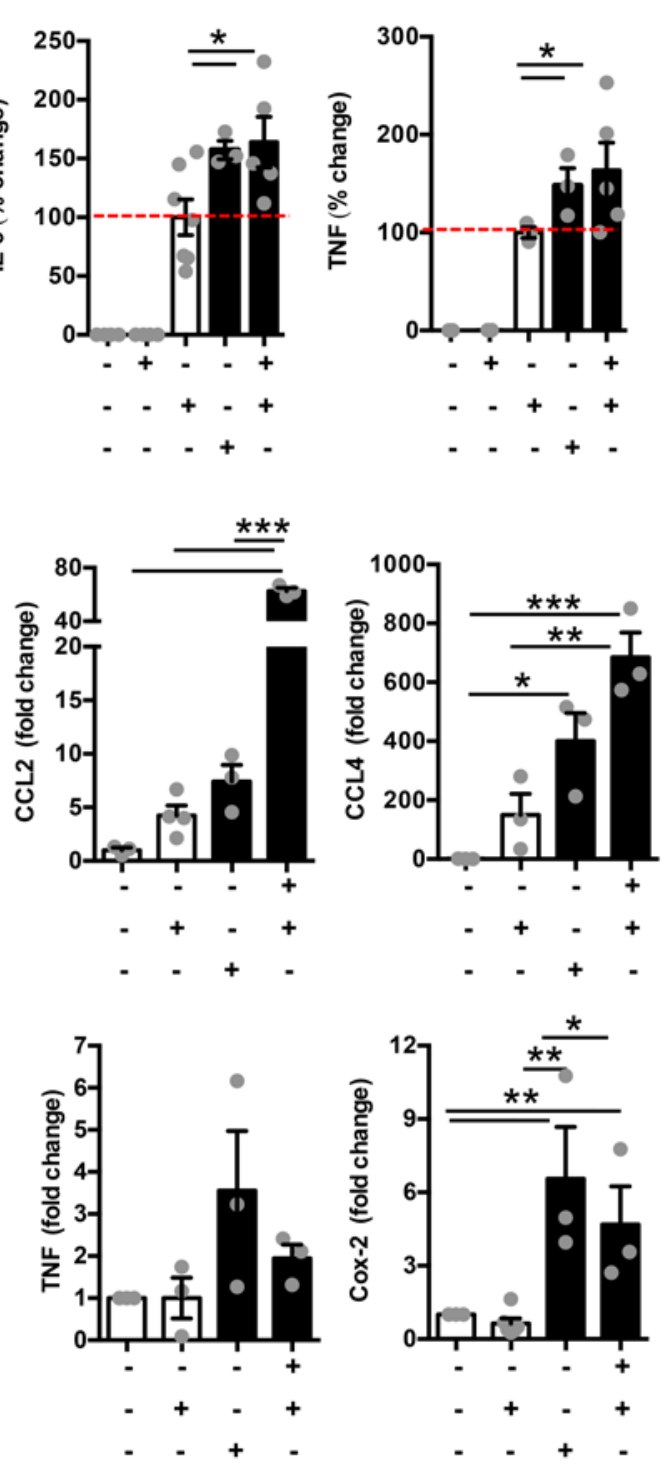
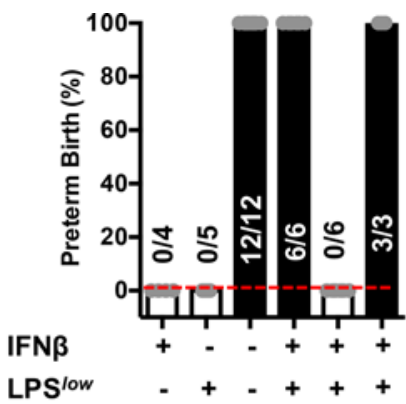

LPS ${ }^{h i g h}-{ }^{-}+-\cdot-$

$\begin{array}{lllllll}\operatorname{alL}-6 & - & - & - & - & + & - \\ \lg G & - & - & - & - & - & +\end{array}$

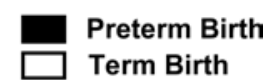

Term Birth
Figure 4. IFN- $\beta$ is sufficient to prime for secondary inflammatory challenge-driven cytokine production and induction of preterm birth (PTB) in mice. (A) A schematic overview of the tractable double-hit preclinical model of PTB induction employed to define the sufficiency of IFN- $\beta$ to prime for secondary inflammatory challenge-driven PTB. Gravid WT mice were treated with IL-6-neutralizing Ab or isotype control Ab (500 $\mu \mathrm{g} /$ mouse) and challenged with recombinant mouse IFN- $\beta\left(10^{4} \mathrm{U} /\right.$ mouse) or LPS alone, or primed with IFN- $\beta$ ( 4 hours) prior to being challenged with LPS ( $25 \mu \mathrm{g} /$ mouse = low; $75 \mu \mathrm{g} /$ mouse $=$ high) on day 16 of gestation and the incidence of PTB was quantified. (B) WT mice ( $n$ $=3-6 /$ condition) were challenged with recombinant mouse IFN- $\beta\left(10^{4}\right.$ $\mathrm{U}$ /mouse) or LPS alone, or primed with IFN- $\beta$ (4 hours) prior to being challenged with LPS (4 hours), and serum IFN- $\beta$, IL-6, and TNF levels were quantified by in vivo cytokine capture assay. (C and D) Gravid WT ( $n=3 /$ condition) mice were challenged with LPS alone or primed with IFN- $\beta$ (4 hours) prior to being challenged with LPS for 12 hours, and uterine CD68, CCL2, CCL4, IL-6, Cox-2, and TNF mRNA expression was quantified. (A) Data represent percentage induction of term birth or PTB. (B) Dashed red line represents $100 \%$ induction of cytokine following LPS ${ }^{\text {low-alone challenge in }}$ WT mice. Data represent percent change over $\mathrm{LPS}^{\text {low }}(\mathrm{WT}) \pm \mathrm{SEM}$. (C and $\mathbf{D})$ Data represent fold change over nonstimulated condition. (B-D) ${ }^{*} P<0.05,{ }^{* *} P<0.01,{ }^{* * *} P$ $<0.001$ by ANOVA followed by Tukey's correction.

was transient in nature, as the effect was abrogated 3 days after the initial priming (Supplemental Figure 8). Overall, these data indicate that IFNAR expression by hematopoietic cells is necessary for IFN- $\beta$ augmentation of LPS-driven proinflammatory cytokine production and likely induction of PTB. These data also argue that such effects represent a transient window of vulnerability, while the sequence of inflammatory events is critical to allow for sensitization to occur.

IFN- $\beta$ priming effects on proinflammatory cytokine production are conserved across species and correlate with PTB in humans. Despite high similarity in immune responses between Mus musculus and Homo sapiens, biological 
A

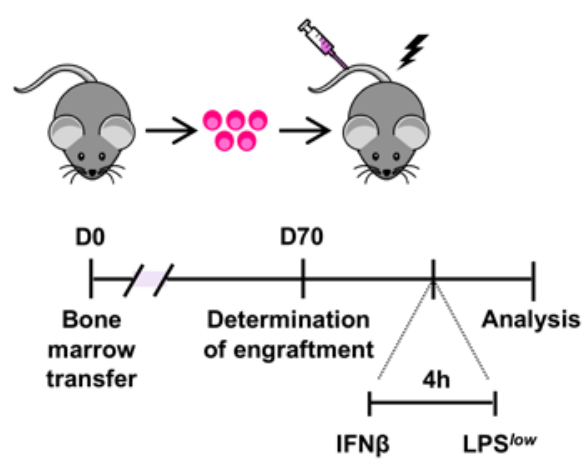

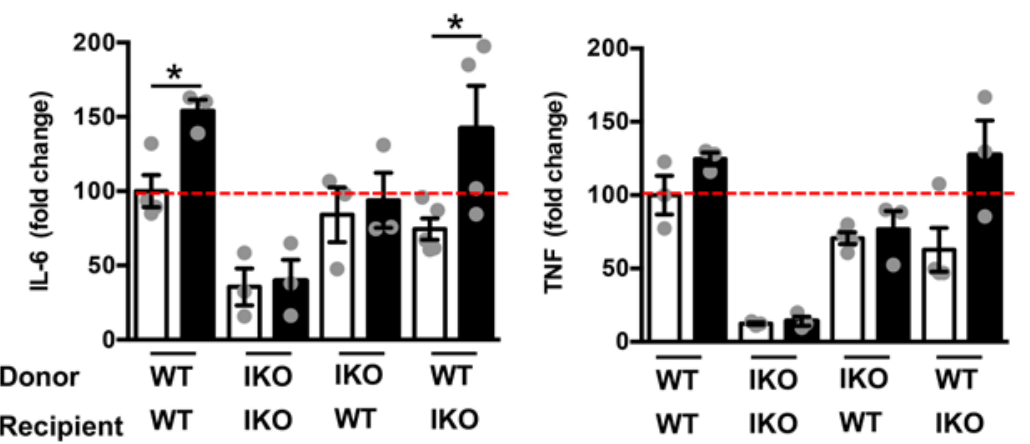

$\square$ LPS $^{\text {low }}$

IFN $\beta+$ LPS $^{\text {low }}$

B
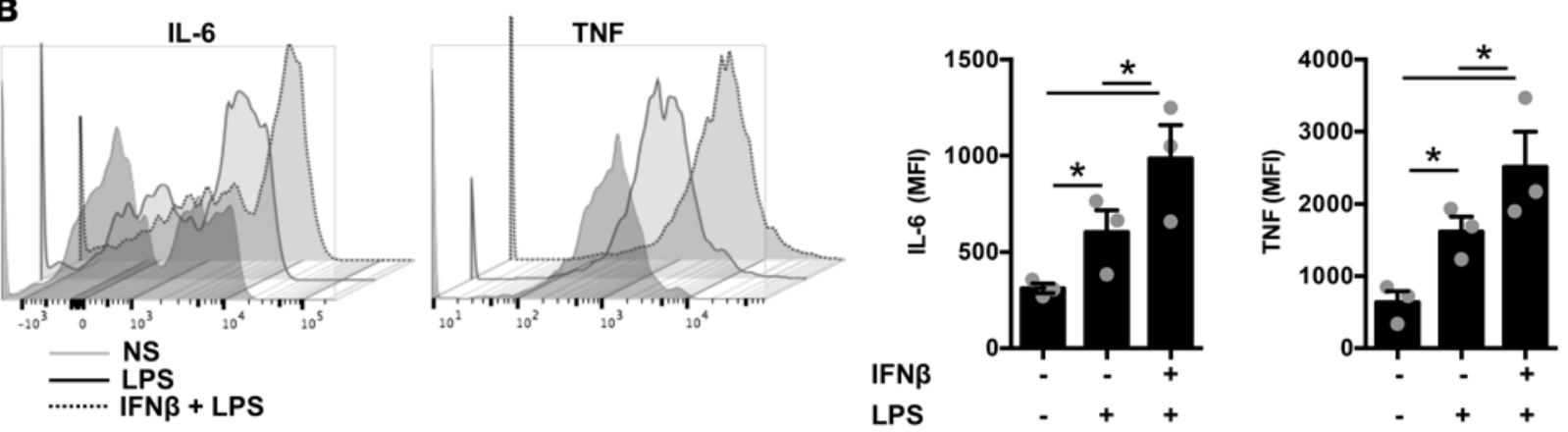

C
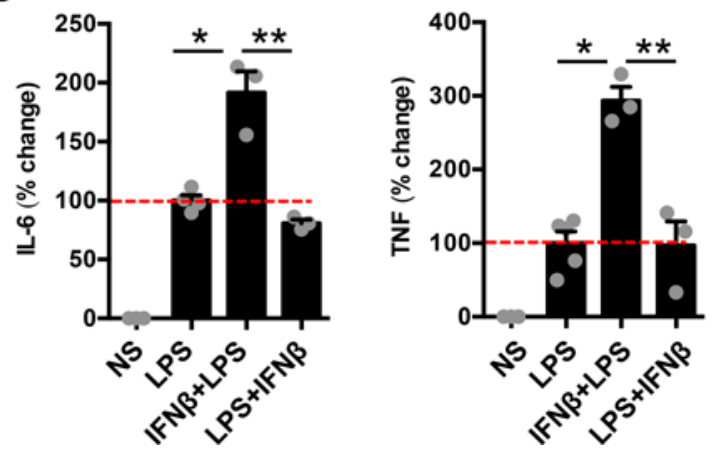

D

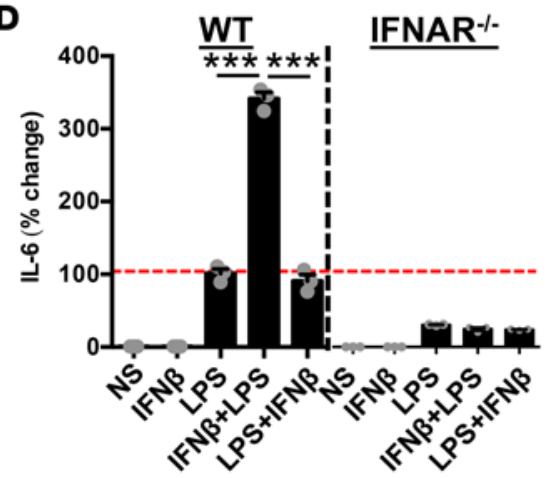

Figure 5. Type I IFN signaling in hematopoietic cells is sufficient for secondary inflammatory challenge-driven cytokine production in mice. (A) Left: A schematic overview of experimental model employed to define the type I IFN receptor-responsive (IFNAR-responsive) cell types sufficient to prime for secondary inflammatory challenge-driven proinflammatory cytokine production. Right: Bone marrow from WT and IFNAR ${ }^{-1-}($ IKO) mice $(n=3-4 /$ condition) was reciprocally transferred into WT and IFNAR ${ }^{-1-}$ recipients and mice were mock treated (saline) or treated with recombinant mouse IFN- $\beta$ (10 ${ }^{4} \mathrm{U} / \mathrm{mouse}$; 4 hours) prior to being challenged with LPS ${ }^{\text {low }}$ ( $25 \mu \mathrm{g}$ /mouse; 4 hours) and serum IL-6 and TNF levels were quantified by in vivo cytokine capture assay (IVCCA). (B) Left: Representative flow cytometry histogram showing modulation of IL-6 and TNF expression after each stimulation. Murine peripheral blood mononuclear cells $(n=3)$ were mock treated or treated with mouse IFN- $\beta(250 \mathrm{U} / \mathrm{ml} ; 4$ hours) prior to being stimulated with LPS (100 ng/ml; 4 hours), and IL-6 and TNF levels were quantified (right) by intracellular flow cytometry. MFI, mean fluorescence intensity; NS, saline. (C) WT mice ( $n=3-4 /$ condition) were challenged with recombinant mouse IFN- $\beta$ (104 U/mouse), LPS ${ }^{\text {low }}$, or IFN- $\beta+$ LPS and vice versa, and serum IL- 6 and TNF levels were quantified by IVCCA. (D) WT and IFNAR ${ }^{-/-}$murine peritoneal macrophages ( $n=3 /$ genotype), were treated as in $\mathbf{B}$ and IL-6 levels were quantified by ELISA. (A, C, and $\mathbf{D}$ ) Dashed red line represents $100 \%$ induction of cytokine following LPS low-alone challenge in WT mice. Data represent percentage change over LPS ${ }^{\text {low }}($ WT) \pm SEM. ${ }^{*} P<0.05,{ }^{* *} P<0.01,{ }^{* *} P<0.001$ by ANOVA followed by Tukey's correction.

processes regulating pregnancy, including gestation length, placentation, and induction of parturition, are different (3). Parturition biology between nonhuman primates and humans, however, is analogous. Thus, we examined whether IFN- $\beta$ priming effects were conserved during nonhuman primate and human pregnancy. Like in mice, IFN- $\beta$ priming augmented LPS-driven IL- 6 and TNF production in PBMCs and decidual cells from pregnant rhesus macaques (Figure 6A). We further extended the relevance of our findings to humans. As observed in rhesus macaques, IFN- $\beta$ priming exacerbated LPS-driven IL- 6 and TNF production from PBMCs, primary monocytes, and decidual cells in humans (Figure 6B). As IL-6 levels in amniotic 
A

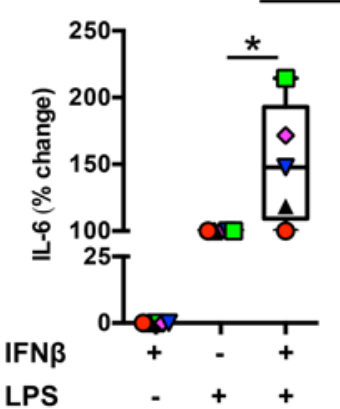

PBMC (R. Macaque)

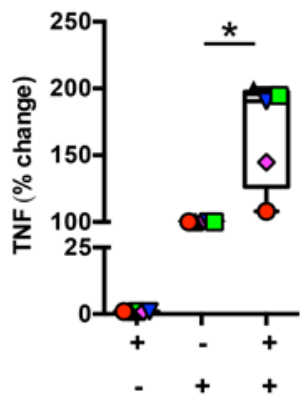

PBMC (Human)

B
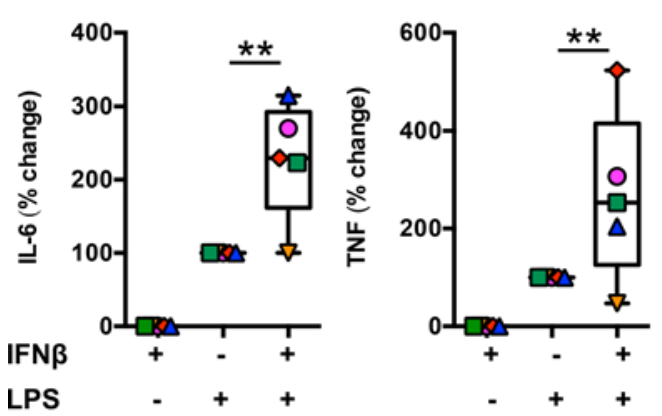

\section{Decidua (Human)}
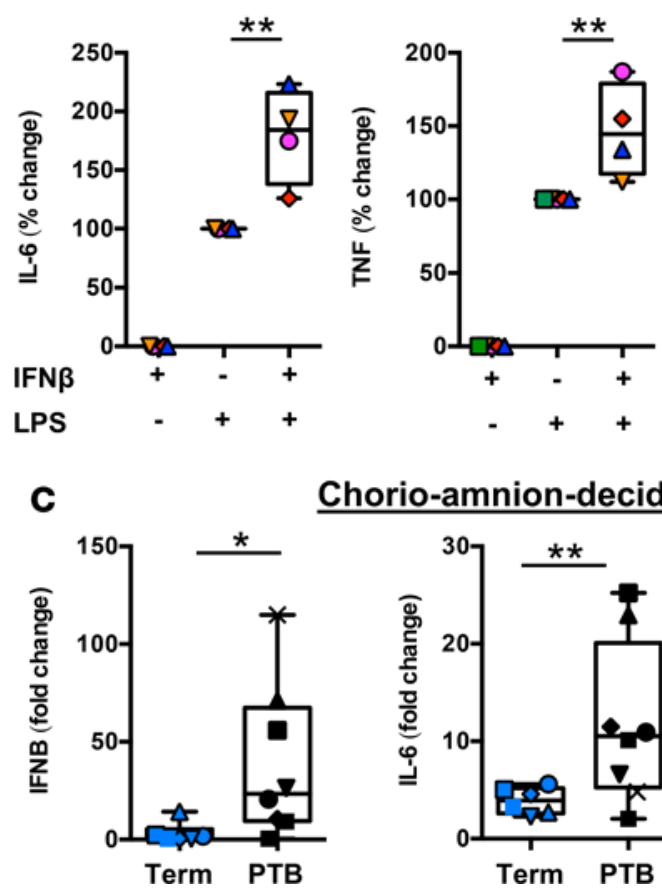

\section{Chorio-amnion-decidua (Human)}
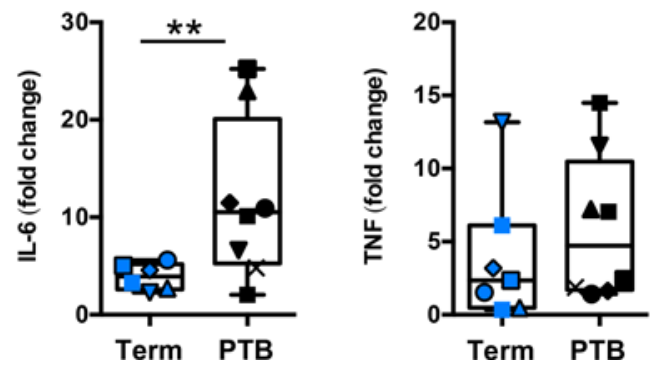

Decidua (R. Macaque)
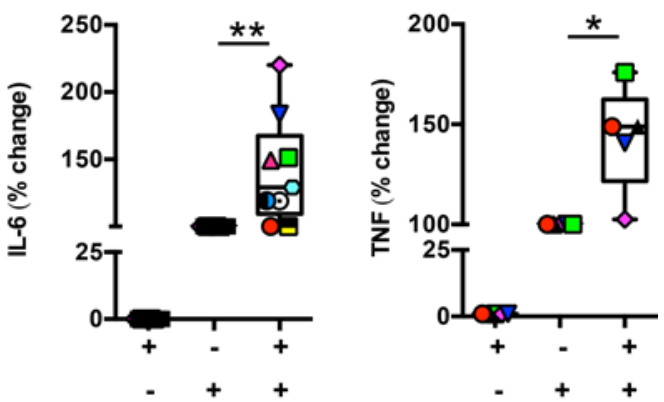

Monocytes (Human)

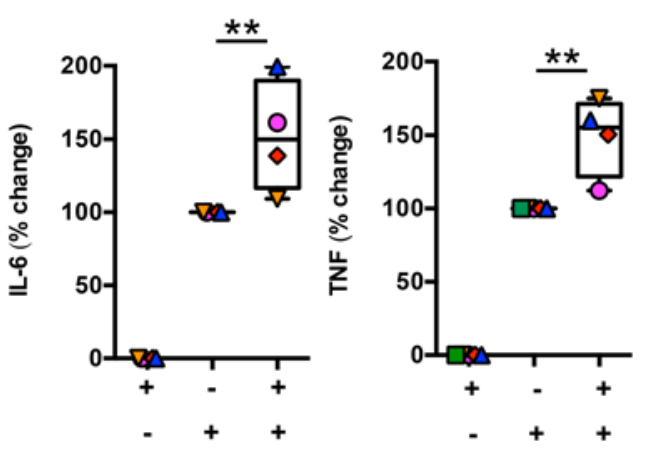

Figure 6. IFN- $\beta$ priming exacerbates secondary inflammatory challenge-driven cytokine production and correlates with preterm birth (PTB) in humans. (A) Peripheral blood mononuclear cells (PBMCs; $n=5$ ) and decidual cells $(n=9)$ obtained from pregnant rhesus macaques were mock treated (saline) or treated with human IFN- $\beta$ ( $250 \mathrm{U} / \mathrm{ml}$; 6 hours) prior to being stimulated with LPS (100 ng/ml; 18 hours), and IL- 6 and TNF levels were quantified by ELISA. (B) PBMCs $(n=5)$ and monocytes $(n=4)$ obtained from healthy human volunteers and decidual cells $(n=4)$ obtained from healthy pregnant human volunteers $(n=4)$ were treated with human IFN- $\beta$ ( $250 \mathrm{U} /$ $\mathrm{ml} ; 6$ hours) prior to being stimulated with LPS $(100 \mathrm{ng} /$ $\mathrm{ml} ; 8$ hours), and IL- 6 and TNF levels were quantified by ELISA. (C) IFN- $\beta$, IL-6, and TNF mRNA expression in the maternal/fetal membranes (chorion-amnion-decidua) from pregnant human volunteers with chorioamnionitis (Term: $n=6$; PTB: $n=8$ ). (A and B) Data represent percentage change over LPS \pm SEM. Significance determined by ANOVA followed by Tukey's correction. (C) Data represent fold change over the average value for chorioamnionitis-negative samples after normalization to the housekeeping 18 S RNA. ${ }^{*} P<0.05,{ }^{* *} P<0.01$ by Mann-Whitney test.

fluid, uterine tissues, and serum $(57,58)$ are thought to be essential for parturition in humans and elevated intraamniotic IL-6 levels have been correlated with PTB risk in humans (59), we also examined whether the same pattern is conserved at the maternal/fetal interface. Importantly, consistent with this observation, increased IFNB, and IL6 expression was observed in human reproductive tissues (chorio-amnion and decidua) from PTB patients with chorioamnionitis (Figure 6C). These data indicate that the type I IFN-mediated regulation of proinflammatory cytokine production is conserved across species and during pregnancy, and might play an important role in the induction of PTB. 


\section{Discussion}

Infection and inflammation are thought to be the leading cause of PTB. Despite the clinical and public health significance, the molecular trigger(s) and immune pathways underlying the pathogenesis of PTB remain underdefined and represent a major gap in knowledge. Our data provide potentially novel insights into the mechanisms regulating the ability of subclinical systemic viral and other infections to augment inflammatory vigor and increase the risk of PTB associated with secondary inflammatory insults. Further, these data shed light on the complex nature of innate immune activation strength, duration, and sequence in pregnancy. The evolutionary advantage of such a biological process is to enable pathogen clearance in the context of polymicrobial infection, which is primarily focused on promoting maternal survival. However, such immune hyperresponsiveness is likely to hold detrimental potential for the offspring due to induction of premature parturition.

In this report, we demonstrate for what we believe is the first time that: (a) the type I IFN/IFNAR axis is critical for sensitization to secondary inflammatory challenge-driven PTB; (b) activation of this axis reduces the threshold needed for induction of PTB through amplification of secondary inflammatory challenge-driven proinflammatory cytokine production including IL-6 and TNF; (c) hematopoietic IFNAR expression is critical for observed hyperresponsiveness; (d) initial type I IFN sensing is required for exacerbation of proinflammatory cytokine production; and (e) these effects are conserved across species and during pregnancy, and thus might play and important role in the induction of PTB in humans.

Infection/inflammation accounts for approximately $40 \%$ of PTB. Timely parturition as well as PTB represents an inflammatory process that includes secretion of various chemokines and cytokines by resident and infiltrating immune cells into reproductive tissues (6). Previous reports have demonstrated that myeloid cells, and macrophages specifically, are central to induction of PTB (46). Notably, macrophage activation exerts its effects on the uterus through the release of inflammatory mediators that drive induction of uterine activation and cervical ripening and induction of parturition (60). Pregnant women, compared with nonpregnant women, are more severely affected by infections, including influenza virus and Listeria (61). However, the impact of a subclinical systemic infection during pregnancy - an infection without an obvious inflammatory component requiring hospitalization — on induction of PTB is not known. Further, whether the above-mentioned mechanisms underlying immune activation and induction of PTB are modulated in the context of a subclinical systemic infection during pregnancy has not been investigated.

Via utility of our double-hit preclinical model we gained potentially novel insights into immune mechanisms central to subclinical viral infection-driven sensitization to secondary bacterial inflammation-induced PTB. Specifically, we show that subclinical infection with viral pathogens (at concentrations markedly lower than those commonly employed to examine primary infection-driven pathological outcomes; see refs. 62, 63) was sufficient to robustly augment secondary inflammatory challenge-driven IFN- $\beta$, IL- 6 , and TNF production and greatly reduce the dose (3-fold) of secondary systemic inflammatory stimuli required for induction of PTB. Further, our findings are supported by recent studies $(64,65)$ showing that placental viral infection, which is known to induce type I IFN production, sensitized animals to bacterial products predisposing to PTB. Overall, these findings demonstrate that the ability of the type I IFN axis to sensitize to PTB is not anatomically restricted and is likely to play an important role in a significant portion of PTB cases. Of interest, however, whether subclinical systemic infection with type I IFN inducing-pathogens can exacerbate responsiveness to common pathogens associated with PTB, that largely colonize the genitourinary tract including group B Streptococcus (66) and other TLR signaling-capable pathobionts (e.g., Ureaplasma) (67), is not known.

Maternal inflammation is physiologically required for uterine contractility, cervical ripening, and the onset of both term birth and PTB. The central role of proinflammatory cytokines, and IL-6 in particular, is supported by previous studies demonstrating the relevance of IL-6 in the regulation of timely parturition in humans (57) and mice (42). Furthermore, elevated IL-6 levels have been correlated with PTB risk in humans (59). Notably, proinflammatory cytokines are known inducers of Cox-2 (60). Cox-2 signaling activation, which is central to prostaglandin production within intrauterine tissues, correlated with induction of PTB in mice and humans $(35,36)$, while Cox-2-selective inhibition prevented preterm delivery (36). Our data suggest that systemic IFN- $\beta$ priming is sufficient to lower the necessary threshold for induction of inflammatory signals not only systemically (e.g., IL-6 and TNF serum levels) but also at reproductive sites (e.g. CCL2, CCL4, CD68, IL-6, TNF, and Cox-2 in uterine tissue), as well as sensitizes for PTB. Type I IFNs 
(in particular, IFN- $\beta$ ) have been shown to regulate macrophage production of proinflammatory cytokines in response to LPS (68). Notably, maternal macrophages constitutively express both IFNAR and TLRs (69) and have been shown to play a central role in TLR9-driven PTB (46). Neonatal blood cells have been shown to exhibit an impaired expression of IFN- $\beta$ and IFN-inducible genes (70), while in the context of viral or intracellular bacterial infection, the majority of systemic type I IFN production is dependent on activation of maternal myeloid-like cells (13). Our data suggest that IFNAR expression by hematopoietic cells is necessary for IFN- $\beta$ priming of exacerbated LPS-driven cytokine production. Although our data and published reports clearly suggest the impact of type I IFN on pathogen-driven inflammatory vigor, such findings also invoke the necessary future areas of investigation that require a more definitive identification of the anatomical locus (intrauterine vs. systemic) and critical cell type(s) (immune cells vs. other cells types) of type I IFN responsiveness in pregnancy and specifically in the context of the double-hit hypothesis.

Whether the effects observed in our report are unique to IFN- $\beta$ or are conserved among all type I IFN family members remains unclear. Our data suggest that IFN- $\alpha$ production, unlike IFN- $\beta$, is not as robustly potentiated in the context of subclinical infection followed by secondary bacterial challenge. The lack of observed potentiation is likely associated with the selective specificity of LPS to induce IFN- $\beta$ production (71) and/or the relative affinities of IFN- $\beta$ and IFN- $\alpha$ subtypes for the 2 IFNAR subunits (IFNAR1 and 2) (55). In fact, IFN- $\beta$ holds a unique ability to specifically interact with IFNAR1 in an IFNAR2-independent manner (55). However, a formal examination of the sufficiency of IFN- $\alpha$ subtypes in the regulation of cytokine production and induction of PTB is required. Similarly, the contribution of IFN- $\varepsilon$, a type I IFN family member that is constitutively expressed by epithelial cells of the female reproductive tract and protects from common sexually transmitted infections (72), should be similarly investigated in the context of pathogen/inflammatory insult-driven PTB. Thus, in-depth analysis of the unique attributes of various type I IFN family members could provide biologically novel, clinically significant, and evolutionarily relevant understandings of the unique ability of type I IFN family members to regulate inflammatory vigor and parturition.

The requirement for the unique sequence of infectious or inflammatory events in dysregulation of proinflammatory cytokine production and induction of PTB remains unknown. Our data suggest that initial type I IFN sensitization is required for dysregulated proinflammatory cytokine production. Although not examined in our report, it is likely that initial infection by viruses or pathogens that drive robust type I IFNs will similarly play a critical role in controlling susceptibility to secondary bacterial challenge. Our data suggest that the ability of type I IFNs to prime for secondary inflammatory challenge is temporally restricted. In fact, these data hint at a limited window of vulnerability of subclinical viral infections in the induction of PTB - something that potentially explains the restricted frequency of PTB cases. Thus, the role of vaccination in limiting pathogen-driven maternal infection/inflammation both systemically and at reproductive sites might be of great importance. In particular, considering the positive correlation of influenza vaccination in pregnant women with protection from PTB (73), it is possible that vaccination reduces the ability of subclinical viral infections to induce type I IFN - something that is associated with humoral immune responses to pathogens after vaccination - and hence reduces the effectiveness or even the transient window of opportunity for sensitization to secondary inflammatory insult-driven PTB.

Multiple mechanisms regulate proinflammatory cytokine production (74). Among these, SUMOylation, a posttranslational modification of protein activity, interactions, and subsequent gene expression, has recently been shown to restrain inflammation through ubiquitin-conjugating enzyme E2 (Ubc9)-dependent repression of transcription factor occupancy of the IFN- $\beta$ promoter and TLRdriven IFN- $\beta$ and proinflammatory cytokine expression (75). Similarly, Cbx4, a SUMO E3 ligase (76), is known to inhibit LPS-induced transcription and to differentially regulate TLR4- versus TLR3-driven gene expression (77). Further, the ability of influenza virus and $L m$ to interfere with SUMOylation has been implicated in infection establishment and/or persistence (78). Both type I IFN and IL-6 signaling activate STAT3 $(79,80)$, while LPS stimulation in myeloid cells is known to induce expression of the transcription factors C/EBP- $\beta$ and NF- $\kappa$ B, which regulate IL-6 production (81). Notably, SUMOylation regulates STAT3-, NF- $\kappa$ B- and C/EBP- $\beta$-dependent cytokine production $(75,82-85)$. Additional studies, however, are required to uncover whether the mechanistic underpinnings of type I IFN-driven modulation of proinflammatory cytokine production occur via modulation of STAT3 or SUMOylation machinery and their downstream impact on transcriptional networks and epigenetic processes in the context of the double-hit hypothesis. 
Lastly, although the biological processes underlying parturition in mice and humans are different, our analysis in nonhuman primates and human PTB patients suggests that the type I IFN-mediated regulation of cytokine production is conserved across species and during human pregnancy. As such, the robustness of our findings also potentially provide perspectives and possible explanations for other paradigms of biological processes in the induction of PTB, including: the role of viral infection, specifically herpes virus (64), in predisposing to PTB; the role of polymicrobial infections (67) in PTB; the role of IFN- $\varepsilon$ (72) in PTB; and the ability of fetal DNA to induce type I IFNs and sensitize to inflammationdriven parturition (86). In fact, our findings argue for a possible definition of type I IFN-driven genes as biomarkers of risk for inflammation-driven PTB and hence as a predictor for pharmacological inhibition of proinflammatory cytokines (e.g., IL-6) (87) in this context.

\section{Methods}

\section{Reagents}

All cell culture reagents were endotoxin-free to the limits of detection of the Limulus amebocyte lysate assay (Lonza) at the concentrations employed. Both TLR ligands [LPS from E. coli 0111:B4 and poly(I:C)] were ultrapure grade (Invivogen).

\section{Mice}

All mice were on a C57BL/6J background and bred in house. Animals were housed in a specific pathogen-free animal facility at Cincinnati Children's Hospital Medical Center (CCHMC) and handled in high-efficiency particulate-filtered laminar flow hoods with free access to food and water.

\section{Rhesus macaques}

Normally cycling, adult female macaques were bred and identified as pregnant using established methods (88). Hysterotomies were performed with delivery of the fetus at $130 \pm 2$ days of gestational age ( $80 \%$ of term gestation) and PBMCs and decidual cell suspensions were isolated and prepared as previously described (89).

\section{Human samples}

PBMCs and monocytes were isolated from healthy adult female volunteers (of reproductive age) and decidual cells were obtained from placenta following elective cesarean section or uncomplicated spontaneous vaginal delivery. Maternal/fetal membrane (chorio-amnion-decidua) samples were collected immediately after birth from women delivering preterm and term. The severity of chorioamnionitis was graded as previously described (90).

\section{Murine pregnancy and procedures}

Female mice were mated with fertile male mice of the same strain. The presence of a vaginal plug was considered day 1 of pregnancy. Parturition events were monitored on days 17-21 and defined as complete delivery of pups.

Challenges and birth outcome. On day 16 of gestation, gravid mice were mock challenged (saline) i.p. or challenged with LPS or poly(I:C) at indicated concentrations and treated with IL-6-neutralizing antibody $\left(500 \mu \mathrm{g} /\right.$ mouse; clone MP5-20F3, BioXCell) as indicated. Exogenous administration of $10^{4} \mathrm{U} / \mathrm{mouse}$ IFN- $\beta$ (PBL Biomedical Laboratories) was performed 4 hours prior to LPS challenge and IL-6-neutralizing antibody administration. PTB was defined as parturition within 24 hours after TLR ligand challenge (all pups deceased). Term birth was defined as parturition on days 19-21 (all pups alive). For quantification of inflammatory mediators at reproductive sites, uterine samples were collected 12 hours after IFN- $\beta$ and/or LPS or poly(I:C) challenge on day 16 of gestation.

Infections. Mice were infected with influenza virus (PR8) by intranasal inoculation with $6 \times 10^{2} \mathrm{PFU}$ on day 14 of gestation; LCMV (Armstrong-13) by i.p. inoculation with $5 \times 10^{4} \mathrm{PFU}$ on day 14 of gestation; or $L m$ (10403S [WT] or 2319 [ $\Delta$ LLO $\Delta$ PLC] ) by i.v. inoculation with $1 \times 10^{2}$ or $1 \times 10^{8} \mathrm{CFU}$, respectively, on day 15 of gestation. All infected mice were subsequently mock challenged (saline) i.p. or challenged with LPS on day 16 of gestation as indicated. Parturition events were defined as above. 


\section{Cytokine quantification}

In vivo. Systemic IL-6 and TNF levels were quantified using in vivo cytokine capture assay (IVCCA) (91). Briefly, biotinylated capture antibodies against IL-6 (clone MP5-32C11) and TNF (clone TN3) (both eBioscience) were injected i.p. 3 hours prior to infectious agent or TLR-ligand challenge, and serum cytokine levels were determined 4 hours later.

Ex vivo and in vitro. Mouse PBMCs were cultured in RPMI supplemented with $10 \% \mathrm{FBS}, 1 \% \mathrm{~L}$-glutamine, and $1 \%$ penicillin/streptomycin with or without human IFN- $\beta$ (250 U/ml) for 4 hours, subsequently mock stimulated or LPS stimulated $(100 \mathrm{ng} / \mathrm{ml})$ for 4 hours, and stained with directly conjugated monoclonal antibodies (all eBioscience): CD11b (clone M1/70), TNF (clone MP6-XT22), IL-6 clone (MP5-20F3), Gr-1 (clone RB6-8C5), CD11c (clone N418), and CD45 (clone 30-F11). Data were collected using a Fortessa II flow cytometer (BD Biosciences) and analyzed by FlowJo software (Tree Star). Human and rhesus macaque PBMCs, monocytes, and decidua cells $\left(1 \times 10^{6}\right.$ cells $)$ were cultured as above with or without human IFN- $\beta(250 \mathrm{U} / \mathrm{ml})$ for 6 hours, subsequently mock stimulated or LPS stimulated $(100 \mathrm{ng} / \mathrm{ml})$ for 18 hours, and cytokine levels were quantified by ELISA (Biolegend).

Murine thioglycollate-elicited peritoneal macrophages (EPMs) were generated using standard protocols (92). EPMs $\left(1 \times 10^{6}\right.$ cells/well) were cultured with or without IFN- $\beta(250 \mathrm{U} / \mathrm{ml})$ for 4 hours and subsequently mock stimulated or stimulated with LPS $(100 \mathrm{ng} / \mathrm{ml})$ or poly(I:C) $(25 \mu \mathrm{g} / \mathrm{ml})$ for 2 hours to determine mRNA expression, or 18 hours to determine cytokine production by ELISA (BD Biosciences).

\section{Type I IFN activity quantification}

Type I IFN activity in mouse serum samples or cell culture supernatants was measured with reference to a recombinant mouse IFN- $\beta$ using an L-929 cell line transfected with an interferon-sensitive luciferase construct as previously described (93). Luciferase activity was measured on a SpectraMax L luminometer (Molecular Devices). IFN- $\beta$ and IFN- $\alpha$ were also quantified using specific ELISA kits (PBL Interferon Source).

\section{Bone marrow transfer}

Bone marrow chimeric mice were generated using 8-week-old WT or IFNAR ${ }^{-1-}$ recipient mice. Briefly, recipient mice were conditioned by whole-body irradiation and were reconstituted via tail vein injection of $5 \times 10^{6}$ bone marrow cells derived from femurs of WT or $\mathrm{IFNAR}^{-/}$mice. Peripheral blood chimerism was assessed by FACS analysis 10 weeks after bone marrow reconstitution, and mice were challenged as indicated.

\section{Gene expression}

For quantification of mRNA expression in murine samples, cells/tissues were homogenized in TRIzol (Invitrogen), and RNA was extracted and cDNA was generated and quantified as previously described (91) using a Light Cycler 480 II (Roche). The following murine primers were utilized: TNF, CCAGACCCTCACACTCAGATCA and CACTTGGTGGTTTGCTACGAC; CD68, AATGATGAGAGGCAGCAAGAGG and CTTCCCACAGGCAGCACAG; CCL2, TGTCTGGACCCATTCCTTCTTG and AGATGCAGTTAACGCCCCAC; CCL4, TCTCTCCTCTTGCTCGTGGC and GAATACCACAGCTGGCTTGGA; TLR4, TGTCATCAGGGACTTTGCTG and TGTTCTTCTCCTGCCTGACA; Cox-2, AATGACCTGATATTTCAATTTTCCATC and ACTGGGCCATGGAGTGGAC; IL-6, TGGTACTCCAGAAGACCAGAGG and AACGATGATGCACTTGCAGA.

For mRNA expression in the human maternal/fetal membranes (chorio-amnion-decidua), cDNA was quantified using human-specific TaqMan gene expression primers (Thermo Fisher Scientific). IL6, Hs00985639_m1; TNF, Hs99999043_m1; IFNB1: Hs01077958_s1 (TaqMan - Applied Biosystems/Fisher Thermo Scientific). Average mRNA values are fold increases over the average value for chorio-negative samples after internal normalization to the housekeeping 18S RNA.

\section{Statistics}

Data were analyzed by ANOVA followed by Tukey's correction, or Mann-Whitney test in Prism 5a (GraphPad Software, Inc.) as appropriate. A $P$ value less than 0.05 was considered significant. In vivo serum cytokine values were normalized to LPS $(25 \mu \mathrm{g})$ challenge as appropriate and expressed as the fold change. In vitro supernatant cytokine levels were normalized to LPS $(100 \mathrm{ng} / \mathrm{ml})$ and expressed as the fold change. All values are represented as means \pm SEM or as percentage of term birth or PTB induction. 


\section{Study approval}

All studies were performed in accordance with the procedures outlined in the NIH Guide for the Care and Use of Laboratory Animals and approved by the CCHMC IACUC. All rhesus macaques (Macaca mulatta) studies were approved by the IACUC at the University of California, Davis. All human studies were performed under CCHMC IRB-approved protocols.

\section{Author contributions}

MC, SV, MJL, VC, PP, TES, ITWH, JWM, DAG, MEMF, CMR, PS, XS, SGK, CAC, SSW, and SD participated in data generation. MC, SV, VC, PP, XS, RK, CLK, CAC, SSW, and SD participated in analysis and interpretation of data. $\mathrm{KH}, \mathrm{EMJ}, \mathrm{DAH}, \mathrm{SPH}$, and SGK provided materials and technical support and participated in critical review of the manuscript. SSW, CAC, CLK, and SD participated in the conception and design of the study and obtained the funding. MC and SD wrote the manuscript. All authors have reviewed the manuscript and approve the final version.

\section{Acknowledgments}

This study was supported, in part, by the CCHMC Perinatal Institute Pilot and Feasibility Award (to S.D.), Burroughs Wellcome Fund Preterm Birth Research grant 1015032 (to S.D.), March of Dimes Prematurity Research Center Ohio Collaborative for an Innovation Catalyst Grant (22-FY16-125 to S.D.), NIH T32AI118697 (to D.A.G.), and the CCHMC Perinatal Infection and Inflammation Collaborative (to C.A.C., S.G.K., S.S.W., and S.D.). The authors thank Sudhansu K. Dey, Louis Muglia, and Stefanie N. Vogel for their critical remarks and advice with study design. We also thank Eleanor N. Fish for providing IFN- $\beta$-deficient mice and Maha Almanan for help with LCMV infections.

Address correspondence to: Senad Divanovic, Division of Immunobiology, Cincinnati Children's Hospital Medical Center, TCHRF - Location S, Room S.5.409, 3333 Burnet Avenue, Cincinnati, Ohio 45229-3039, USA. Phone: 513.636.0286; E-mail: senad.divanovic@cchmc.org.

ITWH's present address is: Division of Rheumatology and Department of Internal Medicine, University of Colorado Denver, Aurora, Colorado, USA.

1. Muglia LJ, Katz M. The enigma of spontaneous preterm birth. N Engl J Med. 2010;362(6):529-535

2. Romero R, Dey SK, Fisher SJ. Preterm labor: one syndrome, many causes. Science. 2014;345 (6198):760-765.

3. Cappelletti M, Della Bella S, Ferrazzi E, Mavilio D, Divanovic S. Inflammation and preterm birth. J Leukoc Biol. 2016;99(1):67-78.

4. Kourtis AP, Read JS, Jamieson DJ. Pregnancy and infection. N Engl J Med. 2014;371(11):1077.

5. Steinhoff MC, MacDonald N, Pfeifer D, Muglia LJ. Influenza vaccine in pregnancy: policy and research strategies. Lancet. 2014;383 (9929):1611-1613.

6. Cappelletti M, Della Bella S, Ferrazzi E, Mavilio D, Divanovic S. Inflammation and preterm birth. J Leukoc Biol. 2016;99(1):67-78

7. Racicot K, et al. Viral infection of the pregnant cervix predisposes to ascending bacterial infection. J Immunol. 2013;191 (2):934-941.

8. Cardenas I, et al. Placental viral infection sensitizes to endotoxin-induced pre-term labor: a double hit hypothesis. Am J Reprod Immunol. 2011;65(2):110-117.

9. Kwon JY, Romero R, Mor G. New insights into the relationship between viral infection and pregnancy complications. $A m J$ Reprod Immunol. 2014;71(5):387-390.

10. Jamieson AM, et al. Role of tissue protection in lethal respiratory viral-bacterial coinfection. Science. 2013;340(6137):1230-1234.

11. Racicot K, Kwon JY, Aldo P, Silasi M, Mor G. Understanding the complexity of the immune system during pregnancy. Am J Reprod Immunol. 2014;72(2):107-116.

12. Robbins JR, Bakardjiev AI. Pathogens and the placental fortress. Curr Opin Microbiol. 2012;15(1):36-43.

13. Stockinger S, et al. Characterization of the interferon-producing cell in mice infected with Listeria monocytogenes. PLoS Pathog. 2009;5(3):e1000355

14. $\mathrm{Ng} \mathrm{CT}$, et al. Blockade of interferon beta, but not interferon alpha, signaling controls persistent viral infection. Cell Host Microbe. 2015;17(5):653-661.

15. Koerner I, Kochs G, Kalinke U, Weiss S, Staeheli P. Protective role of beta interferon in host defense against influenza A virus. J Virol. 2007;81(4):2025-2030.

16. Dienz O, et al. Essential role of IL-6 in protection against H1N1 influenza virus by promoting neutrophil survival in the lung. Mucosal Immunol. 2012;5(3):258-266.

17. Lauder SN, et al. Interleukin-6 limits influenza-induced inflammation and protects against fatal lung pathology. Eur J Immunol. 2013;43(10):2613-2625.

18. García-Ramírez RA, Ramírez-Venegas A, Quintana-Carrillo R, Camarena ÁE, Falfán-Valencia R, Mejía-Aranguré JM. TNF, IL6, and IL1B polymorphisms are associated with severe influenza A (H1N1) virus infection in the Mexican population. PLoS 
ONE. 2015;10(12):e0144832.

19. Paquette SG, et al. Interleukin-6 is a potential biomarker for severe pandemic H1N1 influenza A infection. PLoS ONE. 2012;7(6):e38214

20. Chiaretti A, et al. IL-1 $\beta$ and IL-6 upregulation in children with H1N1 influenza virus infection. Mediators Inflamm. 2013;2013:495848.

21. Hoge J, et al. IL-6 controls the innate immune response against Listeria monocytogenes via classical IL-6 signaling. J Immunol. 2013;190(2):703-711.

22. Cao L, Lawrence DA. Suppression of host resistance to Listeria monocytogenes by acute cold/restraint stress: lack of direct IL-6 involvement. J Neuroimmunol. 2002;133(1-2):132-143.

23. Harker JA, Lewis GM, Mack L, Zuniga EI. Late interleukin-6 escalates T follicular helper cell responses and controls a chronic viral infection. Science. 2011;334(6057):825-829.

24. Seo SH, Webster RG. Tumor necrosis factor alpha exerts powerful anti-influenza virus effects in lung epithelial cells. $J$ Virol. 2002;76(3):1071-1076.

25. Torres D, et al. Membrane tumor necrosis factor confers partial protection to Listeria infection. Am J Pathol. 2005;167(6):1677-1687.

26. Mitchell BF, Taggart MJ. Are animal models relevant to key aspects of human parturition? Am J Physiol Regul Integr Comp Physiol. 2009;297(3):R525-R545.

27. Ratajczak CK, Fay JC, Muglia LJ. Preventing preterm birth: the past limitations and new potential of animal models. Dis Model Mech. 2010;3(7-8):407-414.

28. Riley JK, Nelson DM. Toll-like receptors in pregnancy disorders and placental dysfunction. Clin Rev Allergy Immunol. 2010;39(3):185-193.

29. Romero R, Mazor M, Munoz H, Gomez R, Galasso M, Sherer DM. The preterm labor syndrome. Ann N Y Acad Sci. 1994;734:414-429.

30. Schaefer TM, Desouza K, Fahey JV, Beagley KW, Wira CR. Toll-like receptor (TLR) expression and TLR-mediated cytokine/ chemokine production by human uterine epithelial cells. Immunology. 2004;112(3):428-436.

31. Fichorova RN, Cronin AO, Lien E, Anderson DJ, Ingalls RR. Response to Neisseria gonorrhoeae by cervicovaginal epithelial cells occurs in the absence of Toll-like receptor 4-mediated signaling. J Immunol. 2002;168(5):2424-2432.

32. Schaefer TM, Fahey JV, Wright JA, Wira CR. Innate immunity in the human female reproductive tract: antiviral response of uterine epithelial cells to the TLR3 agonist poly (I:C). J Immunol. 2005;174(2):992-1002.

33. Yellon SM, Ebner CA, Sugimoto Y. Parturition and recruitment of macrophages in cervix of mice lacking the prostaglandin $F$ receptor. Biol Reprod. 2008;78(3):438-444.

34. Norman JE. Preterm labour. Cervical function and prematurity. Best Pract Res Clin Obstet Gynaecol. 2007;21(5):791-806

35. Chang EY, Zhang J, Sullivan S, Newman R, Singh I. N-acetylcysteine prevents preterm birth by attenuating the LPS-induced expression of contractile associated proteins in an animal model. J Matern Fetal Neonatal Med. 2012;25(11):2395-2400

36. Cha J, et al. Combinatory approaches prevent preterm birth profoundly exacerbated by gene-environment interactions. J Clin Invest. 2013;123(9):4063-4075.

37. Hirota Y, Daikoku T, Tranguch S, Xie H, Bradshaw HB, Dey SK. Uterine-specific p53 deficiency confers premature uterine senescence and promotes preterm birth in mice. J Clin Invest. 2010;120(3):803-815

38. Li L, Kang J, Lei W. Role of Toll-like receptor 4 in inflammation-induced preterm delivery. Mol Hum Reprod. 2010;16(4):267-272.

39. Elovitz MA, Wang Z, Chien EK, Rychlik DF, Phillippe M. A new model for inflammation-induced preterm birth: the role of platelet-activating factor and Toll-like receptor-4. Am J Pathol. 2003;163(5):2103-2111.

40. Lyon D, et al. Integrated review of cytokines in maternal, cord, and newborn blood: part I--associations with preterm birth. Biol Res Nurs. 2010;11(4):371-376.

41. Malaeb S, Dammann O. Fetal inflammatory response and brain injury in the preterm newborn. J Child Neurol. 2009;24(9):1119-1126.

42. Robertson SA, et al. Interleukin-6 is an essential determinant of on-time parturition in the mouse. Endocrinology 2010;151(8):3996-4006.

43. Goepfert AR, et al. The Preterm Prediction Study: association between cervical interleukin 6 concentration and spontaneous preterm birth. National Institute of Child Health and Human Development Maternal-Fetal Medicine Units Network. Am J Obstet Gynecol. 2001;184(3):483-488.

44. Chaturvedi V, et al. CXCR3 blockade protects against Listeria monocytogenes infection-induced fetal wastage. J Clin Invest. 2015;125(4):1713-1725

45. de Weerd NA, et al. Structural basis of a unique interferon- $\beta$ signaling axis mediated via the receptor IFNAR1. Nat Immunol. 2013;14(9):901-907.

46. Thaxton JE, Romero R, Sharma S. TLR9 activation coupled to IL-10 deficiency induces adverse pregnancy outcomes. J Immunol. 2009;183(2):1144-1154.

47. Hamilton SA, Tower CL, Jones RL. Identification of chemokines associated with the recruitment of decidual leukocytes in human labour: potential novel targets for preterm labour. PLoS ONE. 2013;8(2):e56946.

48. Murray PJ, Wynn TA. Protective and pathogenic functions of macrophage subsets. Nat Rev Immunol. 2011;11(11):723-737.

49. Dorhoi A, et al. Type I IFN signaling triggers immunopathology in tuberculosis-susceptible mice by modulating lung phagocyte dynamics. Eur J Immunol. 2014;44(8):2380-2393.

50. Blanco JC, Contursi C, Salkowski CA, DeWitt DL, Ozato K, Vogel SN. Interferon regulatory factor (IRF)-1 and IRF-2 regulate interferon gamma-dependent cyclooxygenase 2 expression. J Exp Med. 2000;191(12):2131-2144.

51. O'Brien WF. The role of prostaglandins in labor and delivery. Clin Perinatol. 1995;22(4):973-984.

52. Sun X, Cappelletti M, Li Y, Karp CL, Divanovic S, Dey SK. Cnr2 deficiency confers resistance to inflammation-induced preterm birth in mice. Endocrinology. 2014;155(10):4006-4014.

53. Stockinger S, et al. Production of type I IFN sensitizes macrophages to cell death induced by Listeria monocytogenes. J Immunol. 2002;169(11):6522-6529.

54. O'Riordan M, Yi CH, Gonzales R, Lee KD, Portnoy DA. Innate recognition of bacteria by a macrophage cytosolic surveillance 
pathway. Proc Natl Acad Sci USA. 2002;99(21):13861-13866.

55. de Weerd NA, et al. Structural basis of a unique interferon- $\beta$ signaling axis mediated via the receptor IFNAR1. Nat Immunol. 2013;14(9):901-907.

56. Spaapen RM, et al. Therapeutic activity of high-dose intratumoral IFN- $\beta$ requires direct effect on the tumor vasculature. J Immunol. 2014;193(8):4254-4260.

57. Greig PC, Murtha AP, Jimmerson CJ, Herbert WN, Roitman-Johnson B, Allen J. Maternal serum interleukin-6 during pregnancy and during term and preterm labor. Obstet Gynecol. 1997;90(3):465-469.

58. Orsi NM, Tribe RM. Cytokine networks and the regulation of uterine function in pregnancy and parturition. J Neuroendocrinol. 2008;20(4):462-469.

59. Romero R, et al. Prevalence and clinical significance of sterile intra-amniotic inflammation in patients with preterm labor and intact membranes. Am J Reprod Immunol. 2014;72(5):458-474.

60. Fang X, Wong S, Mitchell BF. Effects of LPS and IL-6 on oxytocin receptor in non-pregnant and pregnant rat uterus. Am J Reprod Immunol. 2000;44(2):65-72.

61. Kourtis AP, Read JS, Jamieson DJ. Pregnancy and infection. N Engl J Med. 2014;370(23):2211-2218.

62. Stamm A, Valentine L, Potts R, Premenko-Lanier M. An intermediate dose of LCMV clone 13 causes prolonged morbidity that is maintained by CD4 ${ }^{+} \mathrm{T}$ cells. Virology. 2012;425(2):122-132.

63. Pan W, et al. Visualizing influenza virus infection in living mice. Nat Commun. 2013;4:2369.

64. Cardenas I, et al. Viral infection of the placenta leads to fetal inflammation and sensitization to bacterial products predisposing to preterm labor. J Immunol. 2010;185(2):1248-1257.

65. Racicot K, et al. Type I interferon regulates the placental inflammatory response to bacteria and is targeted by virus: mechanism of polymicrobial infection-induced preterm birth. Am J Reprod Immunol. 2016;75(4):451-460.

66. Surve MV, et al. Membrane vesicles of group B Streptococcus disrupt feto-maternal barrier leading to preterm birth. PLoS Pathog. 2016;12 (9):e1005816.

67. Payne MS, Bayatibojakhi S. Exploring preterm birth as a polymicrobial disease: an overview of the uterine microbiome. Front Immunol. 2014;5:595.

68. Thomas KE, Galligan CL, Newman RD, Fish EN, Vogel SN. Contribution of interferon-beta to the murine macrophage response to the Toll-like receptor 4 agonist, lipopolysaccharide. J Biol Chem. 2006;281(41):31119-31130.

69. Huynh L, Wang L, Shi C, Park-Min KH, Ivashkiv LB. ITAM-coupled receptors inhibit IFNAR signaling and alter macrophage responses to TLR4 and Listeria monocytogenes. J Immunol. 2012;188(7):3447-3457.

70. Aksoy E, et al. Interferon regulatory factor 3-dependent responses to lipopolysaccharide are selectively blunted in cord blood cells. Blood. 2007;109(7):2887-2893.

71. Gessani S, Belardelli F, Pecorelli A, Puddu P, Baglioni C. Bacterial lipopolysaccharide and gamma interferon induce transcrip tion of beta interferon mRNA and interferon secretion in murine macrophages. J Virol. 1989;63(6):2785-2789.

72. Fung KY, et al. Interferon- $\varepsilon$ protects the female reproductive tract from viral and bacterial infection. Science. 2013;339(6123):1088-1092.

73. Richards JL, et al. Neonatal outcomes after antenatal influenza immunization during the 2009 H1N1 influenza pandemic: impact on preterm birth, birth weight, and small for gestational age birth. Clin Infect Dis. 2013;56(9):1216-1222.

74. Kondo T, Kawai T, Akira S. Dissecting negative regulation of Toll-like receptor signaling. Trends Immunol. 2012;33(9):449-458.

75. Decque A, et al. Sumoylation coordinates the repression of inflammatory and anti-viral gene-expression programs during innate sensing. Nat Immunol. 2016;17(2):140-149.

76. Kagey MH, Melhuish TA, Wotton D. The polycomb protein Pc2 is a SUMO E3. Cell. 2003;113(1):127-137.

77. Amit I, et al. Unbiased reconstruction of a mammalian transcriptional network mediating pathogen responses. Science. 2009;326(5950):257-263.

78. Wimmer P, Schreiner S, Dobner T. Human pathogens and the host cell SUMOylation system. J Virol. 2012;86(2):642-654

79. Ivashkiv LB, Donlin LT. Regulation of type I interferon responses. Nat Rev Immunol. 2014;14(1):36-49.

80. Heinrich PC, Behrmann I, Haan S, Hermanns HM, Müller-Newen G, Schaper F. Principles of interleukin (IL)-6-type cytokine signalling and its regulation. Biochem J. 2003;374(Pt 1):1-20.

81. Rego D, Kumar A, Nilchi L, Wright K, Huang S, Kozlowski M. IL-6 production is positively regulated by two distinct Src homology domain 2-containing tyrosine phosphatase-1 (SHP-1)-dependent CCAAT/enhancer-binding protein $\beta$ and NF- $\kappa \mathrm{B}$ pathways and an SHP-1-independent NF- $\mathrm{B}$ pathway in lipopolysaccharide-stimulated bone marrow-derived macrophages. $J$ Immunol. 2011;186(9):5443-5456.

82. Liu X, Chen W, Wang Q, Li L, Wang C. Negative regulation of TLR inflammatory signaling by the SUMO-deconjugating enzyme SENP6. PLoS Pathog. 2013;9(6):e1003480.

83. Zhou Z, et al. SUMOylation and SENP3 regulate STAT3 activation in head and neck cancer. Oncogene. 2016;35(45):5826-5838

84. Berberich-Siebelt F, et al. SUMOylation interferes with CCAAT/enhancer-binding protein beta-mediated c-myc repression, but not IL-4 activation in T cells. J Immunol. 2006;176(8):4843-4851.

85. Eaton EM, Sealy L. Modification of CCAAT/enhancer-binding protein-beta by the small ubiquitin-like modifier (SUMO) family members, SUMO-2 and SUMO-3. J Biol Chem. 2003;278(35):33416-33421.

86. Phillippe M. Cell-free fetal DNA--a trigger for parturition. N Engl J Med. 2014;370(26):2534-2536.

87. Jones SA, Scheller J, Rose-John S. Therapeutic strategies for the clinical blockade of IL-6/gp130 signaling. J Clin Invest. 2011;121(9):3375-3383.

88. Tarantal AF, et al. Intrapulmonary and intramyocardial gene transfer in rhesus monkeys (Macaca mulatta): safety and efficiency of HIV-1-derived lentiviral vectors for fetal gene delivery. Mol Ther. 2005;12(1):87-98.

89. Presicce $P$, et al. Neutrophil recruitment and activation in decidua with intra-amniotic IL-1beta in the preterm rhesus macaque. Biol Reprod. 2015;92(2):56

90. Redline RW, et al. Amniotic infection syndrome: nosology and reproducibility of placental reaction patterns. Pediatr Dev Pathol. 2003;6(5):435-448.

91. Harley IT, et al. IL-17 signaling accelerates the progression of nonalcoholic fatty liver disease in mice. Hepatology. 
2014;59(5):1830-1839.

92. Divanovic S, et al. Negative regulation of Toll-like receptor 4 signaling by the Toll-like receptor homolog RP105. Nat Immunol. 2005;6(6):571-578.

93. Hoebe K, et al. Identification of Lps2 as a key transducer of MyD88-independent TIR signalling. Nature. 2003;424(6950):743-748. 